\title{
Comparative masticatory myology in anteaters and its implications for interpreting morphological convergence in myrmecophagous placentals
}

\author{
Sérgio Ferreira-Cardoso ${ }^{\text {Corresp., } 1}$, Pierre-Henri Fabre ${ }^{1,2}$, Benoît de Thoisy ${ }^{3,4}$, Frédéric Delsuc ${ }^{1}$, Lionel Hautier ${ }^{\text {Corresp. } 1,2}$ \\ ${ }^{1}$ CNRS, IRD, EPHE, Université de Montpellier, Institut des Sciences de I'Evolution de Montpellier (ISEM), Montpellier, France \\ 2 Mammal Section, Life Sciences, Vertebrate Division, The Natural History Museum, London, United Kingdom \\ 3 Institut Pasteur de la Guyane, Cayenne, French Guiana, France \\ 4 Kwata NGO, Cayenne, French Guiana, France \\ Corresponding Authors: Sérgio Ferreira-Cardoso, Lionel Hautier \\ Email address: sff.cardoso@campus.fct.unl.pt, lionel.hautier@umontpellier.fr
}

Background. Ecological adaptations of mammals are reflected in the morphological diversity of their feeding apparatus, which includes differences in tooth crown morphologies, variation in snout size, or changes in muscles of the feeding apparatus. The adaptability of their feeding apparatus allowed them to optimize resource exploitation in a wide range of habitats. The combination of computer-assisted X-ray microtomography $(\mu-\mathrm{CT})$ with contrast-enhancing staining protocols has bolstered the reconstruction of three-dimensional (3D) models of muscles. This new approach allows for accurate descriptions of muscular anatomy, as well as the quick measurement of muscle volumes and fiber orientation. Ant- and termite-eating (myrmecophagy) represents a case of extreme feeding specialization, which is usually accompanied by tooth reduction or complete tooth loss, snout elongation, acquisition of a long vermiform tongue, and loss of the zygomatic arch. Many of these traits evolved independently in distantly-related mammalian lineages. Previous reports on South American anteaters (Vermilingua) have shown major changes in the masticatory, intermandibular, and lingual muscular apparatus. These changes have been related to a functional shift in the role of upper and lower jaws in the evolutionary context of their complete loss of teeth and masticatory ability.

Methods. We used an iodine staining solution $\left(\mathrm{I}_{2} \mathrm{KI}\right)$ to perform contrast-enhanced $\mu$-CT scanning on heads of the pygmy (Cyclopes didactylus), collared (Tamandua tetradactyla) and giant (Myrmecophaga tridactyla) anteaters. We reconstructed the musculature of the feeding apparatus of the three extant anteater genera using 3D reconstructions complemented with classical dissections of the specimens. We performed a comparative description of the musculature of the feeding apparatus in the two morphologically divergent vermilinguan families (Myrmecophagidae and Cyclopedidae) and compare it to the association of morphological features found in other myrmecophagous placentals.

Results. We found that pygmy anteaters (Cyclopes) present a relatively larger and architecturally complex temporal musculature than that of collared (Tamandua) and giant (Myrmecophaga) anteaters, but shows a reduced masseter musculature, including the loss of the deep masseter. The loss of this muscle concurs with the loss of the jugal bone in Cyclopedidae. We show that anteaters, pangolins, and aardvarks present distinct anatomies despite morphological and ecological convergences. 
1 Comparative masticatory myology in anteaters and its implications for interpreting

2 morphological convergence in myrmecophagous placentals

4 Sérgio Ferreira-Cardoso ${ }^{1}$, Pierre-Henri Fabre ${ }^{1,2}$, Benoît de Thoisy ${ }^{3,4}$, Frédéric Delsuc ${ }^{1}$, and

5 Lionel Hautier ${ }^{1,2}$

6 'Institut des Sciences de l'Evolution de Montpellier (ISEM), CNRS, IRD, EPHE, Université

7 de Montpellier, Montpellier, France.

$8{ }^{2}$ Mammal Section, Life Sciences, Vertebrate Division, The Natural History Museum, London, 9 UK.

10 Institut Pasteur de la Guyane, Cayenne, French Guiana, France.

$11{ }^{4}$ Kwata NGO, Cayenne, French Guiana, France.

13 Corresponding authors:

14 Sérgio Ferreira-Cardoso

15 Place Eugène Bataillon, Montpellier, 34095, France

16 Email address: sergio.ferreira-cardoso@umontpellier.fr

Lionel Hautier

Place Eugène Bataillon, Montpellier, 34095, France

Email address: lionel.hautier@umontpellier.fr 


\section{Abstract}

25 Background. Ecological adaptations of mammals are reflected in the morphological diversity

26 of their feeding apparatus, which includes differences in tooth crown morphologies, variation

27 in snout size, or changes in muscles of the feeding apparatus. The adaptability of their feeding

28 apparatus allowed them to optimize resource exploitation in a wide range of habitats. The

29 combination of computer-assisted X-ray microtomography $(\mu-\mathrm{CT})$ with contrast-enhancing

staining protocols has bolstered the reconstruction of three-dimensional (3D) models of

muscles. This new approach allows for accurate descriptions of muscular anatomy, as well as

the quick measurement of muscle volumes and fiber orientation. Ant- and termite-eating

(myrmecophagy) represents a case of extreme feeding specialization, which is usually

accompanied by tooth reduction or complete tooth loss, snout elongation, acquisition of a

long vermiform tongue, and loss of the zygomatic arch. Many of these traits evolved

independently in distantly-related mammalian lineages. Previous reports on South American

anteaters (Vermilingua) have shown major changes in the masticatory, intermandibular, and

lingual muscular apparatus. These changes have been related to a functional shift in the role

of upper and lower jaws in the evolutionary context of their complete loss of teeth and masticatory ability.

41 Methods. We used an iodine staining solution $\left(\mathrm{I}_{2} \mathrm{KI}\right)$ to perform contrast-enhanced $\mu$-CT

42 scanning on heads of the pygmy (Cyclopes didactylus), collared (Tamandua tetradactyla) and giant (Myrmecophaga tridactyla) anteaters. We reconstructed the musculature of the feeding

44 apparatus of the three extant anteater genera using 3D reconstructions complemented with

45 classical dissections of the specimens. We performed a comparative description of the 
46 musculature of the feeding apparatus in the two morphologically divergent vermilinguan

47 families (Myrmecophagidae and Cyclopedidae) and compare it to the association of

48 morphological features found in other myrmecophagous placentals.

49 Results. We found that pygmy anteaters (Cyclopes) present a relatively larger and

50 architecturally complex temporal musculature than that of collared (Tamandua) and giant

51 (Myrmecophaga) anteaters, but shows a reduced masseter musculature, including the loss of

52 the deep masseter. The loss of this muscle concurs with the loss of the jugal bone in

53 Cyclopedidae. We show that anteaters, pangolins, and aardvarks present distinct anatomies

54 despite morphological and ecological convergences. 


\section{Introduction}

56 The Cretaceous terrestrial revolution and the Cretaceous-Paleogene (K-Pg) mass extinction

57 event are often viewed as milestones in placental mammal evolution (Meredith et al., 2011).

58 These events promoted the opening of terrestrial ecological niches available to placentals,

59 contributing to their morphological diversification (Romer, 1974; Alroy, 1999; Halliday et al.,

60 2019). The adaptation of the placental feeding apparatus likely contributed to this radiation

61 (Price et al., 2012), as mechanical processing of food items is essential to ensure a better

62 energetic intake (Hiiemae, 2000). In mammals, food processing essentially occurs via mastication, which mainly consists of mandibular adduction/abduction (sagittal and coronal planes motion; e.g., Herring \& Scapino, 1973). Mandibular elevation during the adduction is performed by the temporal and the masseter, while transverse movements involve mainly the internal pterygoid (Kendall et al., 1993; Hylander, 2006). Despite the homogeneity of the main complexes of the masticatory apparatus among placental mammals, muscular architecture and proportions vary largely (e.g., Parsons, 1896; Toldt, 1905; Turnbull, 1970). This indicates a wide range of functional disparity associated with both phylogenetic constraints and ecological specialization (Samuels, 2009; Hautier, Lebrun \& Cox, 2012; Fabre et al., 2017; Ginot, Claude \& Hautier, 2018; Kohli \& Rowe, 2019). driver of cranial morphological specialization (Varrela, 1990; Barlow, Jones \& Barratt, 1997; Nogueira, Peracchi \& Monteiro, 2009; Hautier, Lebrun \& Cox, 2012; Klaczko, Sherratt \& Setz, 2016; Maestri et al., 2016). In placental mammals, the evolution of myrmecophagy (antand termite-eating) is a textbook example of morphological convergence driven by diet 
77 (McGhee, 2011). It evolved in three of the four major placental clades (e.g., Springer et al., 78 2013), including Laurasiatheria (pangolins and aardwolf), Afrotheria (aardvark), and

Xenarthra (anteaters and giant armadillo). Morphological convergence associated with myrmecophagy is such that early classifications grouped pangolins, aardvarks, and xenarthrans in the monophyletic Edentata (toothless; Vicq-d'Azyr, 1742; Cuvier, 1798). In these taxa, convergent cranial traits related to ant- and termite-eating include tooth reduction or complete loss, extreme snout elongation, and long extensible tongues (Rose \& Emry, 1993;

Davit-Béal, Tucker \& Sire, 2009; Ferreira-Cardoso, Delsuc \& Hautier, 2019; Gaudin et al., 2020). Additionally, myrmecophagy led to the loss of the ability to chew in anteaters, pangolins, and giant armadillos (Naples, 1999; Davit-Béal, Tucker \& Sire, 2009; Vizcaíno et al., 2009).

Anteaters are a good example of the morphofunctional adaptation to myrmecophagy, with their specialized feeding apparatus musculature (Reiss, 1997; Naples, 1999; Endo et al., 2007, 2017) associated with unique skeletal features such as edentulous jaws, unfused mandibular symphysis, and extremely reduced coronoid and angular processes (with the exception of Cyclopes). South American anteaters (Vermilingua, Xenarthra) consist of ten currently recognized extant species (Reeve, 1940; Wetzel, 1985; Hayssen, 2011; Navarrete \& Ortega, 2011; Hayssen, Miranda \& Pasch, 2012; Gaudin, Hicks \& Di Blanco, 2018; Miranda et al., 2018) that split from their sloth sister-group around 58 million years ago (Gibb et al., 2016). The monogeneric Cyclopedidae comprises the pygmy anteaters (Cyclopes sp.), a group of small arboreal recently described cryptic species (Miranda et al., 2018) feeding solely on ants (Montgomery, 1985; Redford, 1987; Hayssen, Miranda \& Pasch, 2012). The Myrmecophagidae include two ant- and termite-eating genera (Montgomery, 1985; Hayssen, 
100 2011): the semi-arboreal collared anteater (Tamandua tetradactyla) and northern tamandua

101 (Tamandua mexicana), and the terrestrial giant anteater (Myrmecophaga tridactyla; Wetzel,

102 1985; Gaudin \& Branham, 1998; Gibb et al., 2016). Despite their similar diets and prey

103 capture strategies (Montgomery, 1983, 1985), the extreme elongation of the myrmecophagid

104 rostrum and the loss of the jugal bone in cyclopedids are illustrative examples of

105 morphological differences between the two families. Moreover, the Cyclopedidae present

106 several peculiar morphological features such as the strongly curved basicranial/basifacial axis

107 or the plesiomorphic unfused pterygoid bones (Gaudin \& Branham, 1998). Yet, the

108 masticatory musculature of the putatively most diversified anteater family (Miranda et al.,

109 2018) has only been briefly explored (Reiss, 1997). A comparative study of the muscles

110 involved in mastication would be key to assess if the adaptation to myrmecophagy

111 constrained the degree of morphofunctional disparity within the Vermilingua.

112 The unique morphology of anteaters has intrigued early anatomists. Rapp (1852)

113 provided the first description of the myology of the collared anteater (T. tetradactyla), but did

114 not include the head musculature. Owen (1856) and Pouchet (1874) described the limb and

115 head muscles of the giant anteater. Galton (1869), Humphry (1869), and Macalister (1875)

116 studied the myology of the pygmy anteater (C. didactylus), but once again did not consider

117 the head muscles. More recently, Naples (1985a) provided a detailed description of the

118 superficial musculature of the head for all pilosans, including the three anteater genera.

119 However, Reiss (1997) was the first to provide a comprehensive description of the head

120 musculature of the northern tamandua, using the pygmy and giant anteaters mostly for

121 comparisons. Naples (1999) and Endo (2007) provided a thorough description of the

122 masticatory musculatures of the giant anteater. Both authors suggested that the reduced 
123 masticatory muscles reflect a functional shift from the typical adduction/abduction cycle

124 towards a predominantly hemimandibular rotation about the anteroposterior axis (roll). Endo

125 et al. (2017) described the masticatory muscles of the collared anteater, highlighting their

126 similarities with those of the giant anteater. The studies listed above concurred on two main

127 points: i) the masticatory musculature is reduced in all anteaters, when compared to their sloth

128 sister-group or to other placental mammals (Naples, 1985b, 1999), and ii) the modified

129 hyolingual apparatus (protruding elongated tongue) coincides with a functional shift of the

130 masticatory apparatus (roll-dominated mandibular movements).

131 Here, we describe the masticatory, facial-masticatory, and intermandibular muscles in

132 the three anteater genera Cyclopes, Tamandua, and Myrmecophaga (Gaudin \& Branham, 133 1998). We used a combination of traditional and virtual dissections to accurately measure

134 muscular mass and volumes, while reconstructing 3D surfaces based on iodine-enhanced

$135 \mu$ CT-scanning (e.g., Gignac \& Kley, 2014; Ginot, Claude \& Hautier, 2018). Our study aims to

136 provide the first comprehensive description of the masticatory apparatus of the three anteater

137 genera. Finally, we compare our results to existing data from other myrmecophagous

138 placentals (pangolins and aardvark). We hypothesize that the convergent reduction/loss of

139 mastication linked to myrmecophagy was accompanied with similar muscular morphologies. 
141 Materials and methods

\section{Biological sampling}

143 We dissected specimens from the three extant anteater genera: Cyclopes didactylus $(n=2)$;

144 Tamandua tetradactyla $(n=3)$; Myrmecophaga tridactyla $(n=1)$. C. didactylus specimens

145 (M1525_JAG, M1571_JAG) and one specimen of T. tetradactyla (UM-778-N) were alcohol-

146 preserved collection specimens previously fixed in a $10 \%$ formaldehyde solution. $T$.

147 tetradactyla (M3074_JAG) and M. tridactyla (M3023_JAG) were frozen collection

148 specimens. T. tetradactyla specimens correspond to wild roadkills while M. tridactyla was a

149 zoo specimen (M3023_JAG). M3075_JAG (T. tetradactyla) was immediately dissected after

150 collection along the road. All heads were extracted and, when possible, the complete sternum

151 and the tongue musculature were also detached (M1525_JAG, M3075_JAG). Frozen and

152 fresh heads were then fixed in a $10 \%$ formaldehyde solution to allow for long term storage.

153 All specimens were stored in 70\% ethanol. All wild specimens were collected in French

154 Guiana and were stored in the collections of the Association Kwata (JAGUARS collection,

155 Cayenne, France) and the Université de Montpellier (UM; Montpellier, France).

157 Conventional dissections

158 For each specimen, only one side was dissected. The areas of insertion and origin were

159 described and each muscle was then stored separately in a 70\% ethanol solution. All muscles

160 were posteriorly removed from the ethanol solution and weighted with a Sartorius A $120 \mathrm{~S}$

161 precision weighing scale (precision $=0.01 \mathrm{mg}$ ). Individual wet muscle masses are provided as 
162 Supplemental Tables. Muscular volume was calculated for the three stained specimens based 163 on mass and a density of 1.06 g.cm ${ }^{-3}$ (Murphy \& Beardsley, 1974). These estimations were

164 then compared to the volumes obtained with the digital segmentations. All dissected

165 specimens were re-stored in a 70\% ethanol solution for a period no longer than two weeks, 166 prior to staining (see below).

167

168

\section{lodine-enhanced CT-scanning}

169

170

171

172

173

174

175

176

177

178

179

180

181

182 183

For each species, the most complete and well-preserved specimen (Fig. S1A-C) was selected to be stained. Contrast-enhanced $\mu \mathrm{CT}$-scans result in an increase of density of the soft tissues and thus the contrast between muscles and bone is lost (Cox \& Jeffery, 2011). Therefore, the specimens were $\mu \mathrm{CT}$-scanned prior to staining, so that the bone tissue could be easily reconstructed. A second scan was performed after staining (see below). High-resolution microtomography $(\mu \mathrm{CT})$ was performed at Montpellier Rio Imaging (MRI; Microtomograph RX EasyTom 150, X-ray source 40-150 kV) platform. Original voxel sizes were $35.0 \mu \mathrm{m}$ for C. didactylus (M1571_JAG), $76.0 \mu \mathrm{m}$ for T. tetradactyla (M3075_JAG), and 112.1 $\mu \mathrm{m}$ for M. tridactyla (M3023_JAG).

The contrast enhancement protocol was adapted from (Cox \& Jeffery, 2011). All specimens were removed from the $70 \%$ ethanol solution and directly transferred to a solution of iodine $\left(5 \% \mathrm{I}_{2} \mathrm{KI}\right)$ for a period of two to eight weeks, depending on size. This concentration represents a trade-off between observed staining efficiency and the soft-tissue shrinkage associated with iodine staining, even if incubation period seems to have a limited effect in soft-tissue shrinkage, after the first two days (Vickerton, Jarvis \& Jeffery, 2013). In $T$. 
184 tetradactyla and $M$. tridactyla, small volumes of $\mathrm{I}_{2} \mathrm{KI}$ solution were directly injected into the 185 muscles, as the large size of the specimens hinders an efficient passive diffusion of the 186 contrasting agent. binning was performed in order to allow for a better handling of the three-dimensional (3D) volumes. 3D volumes of each muscle were generated using Avizo 9.7.0 (Thermo Fisher

Scientific). We generated surfaces for the skull and muscles separately and then used the function "register" in Avizo 9.7.0 to align these reconstructions. Most tendons and aponeuroses were not stained by the iodine solution, and were therefore not reconstructed.

Some muscles may thus appear artificially detached from the skull (e.g., M. masseter superficialis in myrmecophagids).

\section{Nomenclature}

We used the muscular nomenclature for the masticatory apparatus of xenarthrans defined by

Naples (1985a,b, 1999). More recent descriptions of the masticatory apparatuses of $M$. tridactyla and T. tetradactyla adopted the English version of the same terminology (Endo et al., 2007, 2017). All muscle names are fully written in Latin. We follow Naples (1999) in using the term 'pars' to address myologically distinct units with developmentally common origins (e.g., M. buccinatorius pars externa vs M. buccinatorius pars interna), while the term 'pars reflexa' is used here to characterize a part of a myological unit which wraps around a bone structure (e.g., Cox \& Jeffery, 2015). Muscle abbreviations are provided in Table 1. 


\section{Results}

208 Measurements of the muscles involved in mastication are summarized in Tables 2 and 3.

209 Volume measurements were performed on the segmented muscles of the contrast-enhanced

210 specimens. Mass measurements of all dissected specimens are provided in Table S1. Volumes

211 estimated from muscle weights are correlated with those obtained from the 3D-

212 reconstructions for the three specimens (all $p<0.05$; Table S2). In C. didactylus and $T$.

213 tetradactyla the volumes estimated from the mass were smaller than those obtained from 3D-

214 reconstructions, while in $M$. tridactyla they were larger, possibly due to soft tissue shrinking

215 caused by the long period of staining of the latter (eight weeks; Hedrick et al., 2018). Below,

216 we provide an anatomical description of the musculature of each of the three anteater species.

217 The description is followed by a comparative anatomy section in which muscle shape, size

218 (Tables 2 and 3), and architecture are compared among anteaters. Anatomical structures of the

219 skull and mandible that are relevant to the description are depicted in Fig. 1. The origins and

220 insertions of the masticatory muscles are figured for one species (i.e. Tamandua tetradactyla)

221 (Fig. 2) to serve as a reference and to complement the 3D reconstructions. Three-dimensional

222 surface models of the illustrated specimens (Fig. S1) are freely available at

223 MorphoMuseumM (www.morphomuseum.com; Ferreira-Cardoso et al., 2020). 


\section{Anatomical description}

\section{Cyclopes didactylus}

\section{Masticatory apparatus}

\section{M. masseter superficialis}

228 The M. masseter superficialis (M.m.s.; Fig. 3) is the only muscle of the masseter muscle 229 complex present in $C$. didactylus. The M.m.s. is anteroposteriorly elongated and originates

230 from the lateral surface of the zygomatic process of the maxilla (Fig. 1A). The jugal bone is 231 absent in C. didactylus. The origin of the M.m.s. consists of a long and strong posteroventrally projecting tendon that covers the most anterior half of the M.m.s. The muscle fibers of this anterior part are slightly obliquely oriented and compose the pars anterior of the M.m.s. (pa-M.m.s.; Fig. 3). The pa-M.m.s. inserts laterally from the posterior part of the dentary pad (Fig. 1B) to the anterior margin of the condyle (Fig. 3B). The pa-M.m.s. presents a pars reflexa inserting on the ventromedial margin of the ascending ramus of the mandible extending anteroposteriorly from the anterior margin of the coronoid process to the level of the mandibular canal. This part is covered laterally by the tendon from which it originates. Posteriorly, the M.m.s. presents a distinct pars posterior (pp-M.m.s.; Fig. 3; Fig. S2A) with anteroposteriorly oriented fibers. The pp-M.m.s. shares the origin with the pa-M.m.s. The

241 former covers the pa-M.m.s. posteriorly to the coronoid process and inserts on the angular 242 process of the mandible (Fig. 3). Its pars reflexa is continuous with the pars reflexa of the pa243 M.m.s. and almost reaches the most posterior point of the angular process. 


\section{M. masseter profundus}

246 The M. masseter profundus is absent in C. didactylus.

\section{M. temporalis superficialis}

The M. temporalis superficialis (M.t.s.; Fig. 3) is the largest of the four muscles of the dorsal edge of the temporal fossa (Fig. 1 A, area in green). The temporal crest runs from the posterior end of the orbital ridge to the anterior surface of the root of the zygomatic process of the squamosal. A thick tendinous layer stretches from the origin of the M.t.s. and covers the posterodorsal part of the muscle. The M.t.s. is thinner at its origin and thicker at its insertion. The insertion is muscular on the dorsal tip and the dorsal part of the posterior margin of the coronoid process. An aponeurosis runs dorsoventrally along the anterior surface of the M.t.s. and completely covers the lateral and anterior surfaces of the coronoid process. The fiber fascicles of the M.t.s. are organized in a bipennate structure (Fig. S2B). Deep fibers are dorsomedially oriented while superficial ones are dorsolaterally oriented. In cross-section, the insertion angle of medial fibers with the axis of pennation is about $26^{\circ}$, while lateral fibers

261 present an angle of around $12^{\circ}$.

\section{M. temporalis superficialis pars zygomatica}

263 The M. temporalis superficialis pars zygomatica (pz-M.t.s.; Fig. 3A,C) is a relatively small muscle, which is well separated from the M.t.s.. It originates from the ventromedial part of the zygomatic process of the squamosal and broadens ventrally to end on an anteroposteriorly 
266 elongated muscular insertion. The insertion occupies the lateral part of the mandibular notch.

267 The pz-M.t.s. is wider dorsally and thinner ventrally, with fibers presenting an oblique 268 orientation.

\section{M. temporalis profundus pars lateralis}

271 The temporal complex includes a deep component divided in two parts, the M. temporalis

272 profundus pars lateralis (pl-M.t.p.; Figs. 3B and 4A) being the largest. The pl-M.t.p. takes its

273 origin on a pseudo-elliptical area that extends from the posteroventral part of the orbital

274 contribution of the frontal to the anteroventral part of the temporal fossa. The insertion of the

275 pl-M.t.p. covers most of the posterolateral surface of the coronoid process, and narrows

276 posteriorly along the mandibular notch. Contrary to the M.t.s., the pl-M.t.p. does not present a

277 pennate structure, with fibers roughly vertically oriented.

\section{M. temporalis profundus pars medialis}

The M. temporalis profundus pars medialis (pm-M.t.p.; Fig. 4A) consists of the inner part of the M.t.p. that takes its origin from the orbit, between the ventral edge of the temporal fossa and the optic foramen. The pm-M.t.p. and the pl-M.t.p. are clearly separated posteriorly on the insertion, with the posterior tip of the pm-M.t.p. occupying a more ventromedial position at the level of the mandibular foramen. Fiber orientation and shape of the pm-M.t.p. is similar to that of pl-M.t.p., but the former's volume is about two thirds that of the latter. However, both muscles are anastomosed anteriorly. 


\section{M. pterygoideus externus pars superior}

289 The M. pterygoideus externus pars superior (ps-M.p.e.; Figs. 4B and 5A) is a small

290 anteroposteriorly elongated muscle. The ps-M.p.e. arises from a fossa that extends from the

291 ventral part of the parietal, at the lower limit of the temporal fossa, into the glenoid fossa. It is

292 the only part of the pterygoid muscle complex that takes its origin outside the pterygoid fossa.

293 The muscle is mediolaterally compressed and obliquely oriented. Its posterior part presents a

294 small torsion anterior to its ventrolateral projection towards the mandible. The insertion of the 295 ps-M.p.e. consists of a small concavity just medioventral to the head of the articular condyle.

\section{M. pterygoideus externus pars inferior}

298

299

300

301

302

303

304

305

306

307

308

309

The pars inferior of the M.p.e. (pi-M.p.e.; Figs. 3B and 4A) consists of a short and fleshy muscle strap. The pi-M.p.e. originates from a small area on the sphenoid, laterally to the foramen rotundum, and dorsally adjacent to the origin of the M. pterygoideus internus. The muscle is mediolaterally wide and presents a more horizontal orientation than the ps-M.p.e.. The pi-BE projects posterolaterally to insert on the anterior margin of the articular condyle, at mid-height. The medial part of the pi-M.p.e. projects posteriorly, inserting below the insertion area of the ps-M.p.e., reaching the mid-length of the head of the condyle.

\section{M. pterygoideus internus}

The M. pterygoideus internus (M.p.i.; Figs. 3, 4, and 5) arises from the pterygoid fossa and consists of a fleshy block that originates from the posterolateral part of the palatine to the level of the anterior margin of the ectotympanic (Fig. 3A, B). Its fibers run anteroposteriorly 
310 with an oblique orientation and insert medially on the angular process of the mandible, from

311 the level of the anterior margin of the head of the articular condyle to its posterior margin. In

312 the most posterior part of their insertion, the fibers have a more posteroventral direction and

313 form a small pars reflexa that wraps the posteriormost tip of the angular process. A dense

314 connective tissue lies dorsal to the insertion of the M.p.i., posterior to the opening of the

315 mandibular canal.

\section{Facial-masticatory musculature}

318

319

320

321

322

323

\section{M. buccinatorius pars externa}

The M. buccinatorius pars externa (pe-M.b.; Fig. 3A,C,D) is distinguishable from the internal part of this muscle. It is a sheet-like muscle that envelopes the external surface of the $M$.

buccinatorius pars interna, as well as the buccal salivary glands. Its origin stretches along the ventral edge of the maxilla and the palatine, from anteriorly to the inferior orbital foramen until the anterior part of the insertion of the M. pterygoideus internus. The ventral part of the pe-M.b. wraps the ventral portion of the M. buccinatorius pars interna (and the salivary glands, anteriorly) and attaches on a broad insertion area on the lateral surface of the mandible. The fibers have a dorsoventral orientation.

\section{M. buccinatorius pars interna}

The pars interna of the M. buccinatorius muscle (pi-M.b.; Fig. 3B) is more voluminous when compared to the pars interna. The pi-M.b. originates from a thin fiber bundle posterior to the buccal commissure and is covered by the pe-M.b. just posteriorly. The pi-M.b. is bordered by 
332 the salivary glands, ventrally and laterally, anterior to the level of the sphenopalatine foramen.

333 The pi-M.b. is a long muscle that reaches as far posteriorly as the level of the coronoid

334 process. It is characterized by a buccal projection that sits between the upper and lower jaws

335 (Fig. 3D). The lateral part of the pi-M.b. contacts the pe-M.b. and does not attach to any bone

336 surface. Posteriorly, the pi-M.b. inserts on the dorsomedial surface of the mandible, along the

337 fossa located between the posterior part of the dentary pad and the coronoid process. Its

338 insertion ends posterior to the coronoid process where it contacts the M. temporalis profundus

339 pars medialis and the anterior part of the M. pterygoideus internus. The fibers of the pi-M.b.

340 are anteroposteriorly oriented.

\section{M. mandibuloauricularis}

343 The M. mandibuloauricularis (M.m.a.; Fig. 3A, C, and D) is a strap-like bundle that takes its 344 origin on the anteroventral part of the auricular cartilage. The M.m.a. projects ventromedially 345 to insert on the posterodorsal edge of the angular process of the mandible. The insertion is 346 small and is located between the posterior parts of the masseteric and pterygoid fossae of the 347 mandible. The M.m.a. fibers presents a mediolateral orientation with a strong ventral 348 component. 


\section{M. intermandibularis anterior}

352 The M. intermandibularis anterior (M.i.a.; Fig. 5) is a thin, dorsolaterally wide, and elongated

353 muscle. Naples (1999a) described this muscle as the anterior part of the M. mylohyoideus pars

354 anterior. The M.i.a. takes its origin on the cartilage of the unfused mandibular symphysis.

355 The muscle has two insertions on the ventrolateral margin of both hemimandibles, wrapping 356 around their ventral edges. In ventral view (Fig. 5B), it covers the anterior part of the base of

357 the tongue and the anterior part of the geniohyoideus (Fig. 5). The M.i.a. extends posteriorly 358 for about half the length of the mandible, its posterior end being clearly separated from the

359 anterior margin of the M. mylohyoideus pars anterior (see below). Its fibers are transversely

360 oriented and are continuous between mandibles, with this muscle consisting of one single 361 element.

\section{M. mylohyoideus pars anterior}

364

365

366

367

368

369

370

The M. mylohyoideus pars anterior (pa-M.mh.; Fig. 5) consists of a fibrous sheet that originates ventrally to the dentary pad, on the medial surface of the mandible. This muscle is homologous to the pars medius of the M. mylohyoideus described by Naples (1999a). The origin area stretches from the widest point of the dentary pad to its posteriormost point. Posteriorly, its origin shifts from the mandible to the ventromedial surface of the $M$. pterygoideus internus (M.p.i.). At the posterior end of the M.p.i. the origin changes again, creating a dorsolateral gap separating the anterior and the posterior fibers. We consider this to 
371 be the posterior limit of the pa-M.mh., with the posterior part being considered the $M$.

372 mylohyoideus pars posterior. The fibers are transversely oriented ventrally and insert along a

373 fibrous midline raphe that connects the left and right pa-M.mh.s (Fig. S2C).

\section{M. mylohyoideus pars posterior}

The M. mylohyoideus pars posterior (pp-M.mh.; Fig. 5) is continuous with the pa-M.mh. The division between the two parts is set by the difference of the origin. The pp-M.mh. takes its origin on the ventromedial surface of the tympanic bulla, parallel to the auditory tube. The fibers display the same orientation as in the pars anterior and insert on a fibrous midline raphe. However, near the posterior end of the hard palate, the left and right muscles appear to anastomose in the midline, with the intertonguing contact becoming less spaced. As the interstylohyoideus (Fig. 5) and the posterior part of the M. mylohyoideus pars posterior were not preserved in our specimens of $C$. didactylus, the attachment of the pp-M.mh. to the hyoid system is not visible.

\section{$\underline{\text { Tamandua tetradactyla }}$}

Masticatory apparatus

\section{M. masseter superficialis}

The M.m.s. (Fig. 6) is a fleshy, anteroposteriorly long muscle; its anterior and posterior ends are angular in shape in lateral view. The fibers of the M.m.s. are slightly oblique and take 
391 their origin on the lateral surface of the zygomatic process of the maxilla through a strong

392 tendon. The M.m.s. inserts on the shallow masseteric fossa of the mandible. It covers most of

393 the lateral surface of the ascending ramus, including most of the more anterior M. masseter

394 profundus (see below). The M.m.s. is thicker posteriorly, and thins down anteriorly as it

395 overlies the M. masseter profundus. The tendon of the M.m.s. was not visible in the contrast-

396 enhanced specimen. The M.m.s. presents a pars reflexa that runs from the level of the

397 posterior part of the jugal to the posterior tip of the angular process of the mandible.

398 Anteriorly, the M.m.s. presents a small projection towards the zygomatic process of the 399 mandible.

400

401

\section{M. masseter profundus}

402

The M. masseter profundus (M.m.p.; Figs. 4B and 6) is smaller than its superficial counterpart

403 (M.m.s.). It takes its origin on the anterior part of the ventromedial surface of the jugal bone. Anteriorly, its origin area includes the most posteroventral surface of the zygomatic process 405 of the maxilla. The fibers of the M.m.p. run obliquely to insert posteroventrally on the lateral 406 surface of the mandible. The fibers are more vertical than those of the M.m.s. The muscle presents components with slight lateral and anterior orientations. The insertion area on the mandible stretches from the coronoid process to the level of the oblique line. Contrary to the M.m.s., the M.m.p. is thicker at its origin than at its insertion. 
411

412 413 largest, arising from a relatively large surface between the dorsal edge of the temporal fossa

\section{M. temporalis superficialis}

The M.t.s. (Fig. 6) is one of the three muscles that forms the temporal complex. It is also the
415

416

417

418

419

420

421

422

423

424

425

426

427

428

429

430

431

432 and the origin of the ps-M.p.e. (Fig. 6). It is wide and broad in lateral view, and transversely compressed. It presents a fan-like shape, the fibers converging ventrally towards the small and flat coronoid process. The M.t.s. is medial to a large lacrimal gland, which fills most of the temporal fossa. The lateral surface of the M.t.s. is covered by a thin tendinous layer. Ventrally, the M.t.s. inserts on the dorsomedial surface of the coronoid process via a large aponeurosis. The M.t.s. muscle fibers are oriented vertically in the anterior part of the muscle, and are more oblique posteriorly.

\section{M. temporalis superficialis pars zygomatica}

The pars zygomatica of the M.t.s. (pz-M.t.s.; Fig. 6) is a small fleshy strip on the ventral margin of the M.t.s. Unlike the M.t.s., the pz-M.t.s. originates on a small area limited to the ventral surface of the zygomatic process of the squamosal (Fig. 6). Its obliquely oriented fibers insert on the dorsolateral surface of the mandibular notch. While the insertion area and orientation of the fibers are distinct from the anterior part of the M.t.s., both muscles are anastomosed posteriorly to their mid-length.

\section{M. temporalis profundus pars lateralis}

The M.t.p. (Fig. 4B and 6) is divided into two distinct parts. The pars lateralis (pl-M.t.p.) is a small fleshy block deep to the larger M.t.s. The pl-M.t.p. takes its origin from the crest formed 
433 between the anteroventral border of the temporal fossa and the groove for the ophthalmic vein

434 and the oculomotor nerve (III) (orbital process). The pl-M.t.p. transversely widens from its

435 origin to its insertion. Fiber orientation is similar to that of the anterior part of the M.t.s.,

436 although slightly more oblique in coronal view. The insertion of the pl-M.t.p. is short and

437 extends from the mid-length of the mandibular notch to the anterior part of the coronoid

438 process. It covers most of the dorsal surface of the mandible in width. While the insertion is

439 mostly muscular, the pl-M.t.p. shares the aponeurosis with the M.t.s. anteriorly.

\section{M. temporalis profundus pars medialis}

442 The pm-M.t.p. (Fig. 4B and 6) is the smallest part of the temporal muscle complex. It has no 443 insertion, as it anastomoses with the pl-M.t.p. posterolaterally, but both parts could be easily 444 separated during dissection. The fibers of the pm-M.t.p. are vertically oriented. Their insertion 445 is medial to that of the pl-M.t.p. and extends from the level of the anterior tip of the pi-M.p.e. 446 to the anterior margin of the optic foramen. The medialmost part of the pm-M.t.p. wraps the 447 mandible medially to insert on its dorsomedial surface; it contacts the dorsal part of the $M$. pa448 M.mh. (see 'Intermandibular musculature').

\section{M. pterygoideus externus pars superior}

451 The ps-M.p.e. (Fig. 4B and 6B) is a strap-like muscle that arises from an elongated fossa 452 along the ventral limit of the temporal fossa. Its obliquely oriented fibers run posteriorly to 453 medially wrap around the head of the articular condyle of the mandible (Fig. 4B). The 
454 insertion extends from the anterior part to the posterior tip of the blunt articular condyle. The 455 ps-M.p.e. overlies the insertion of the pi-M.p.e. (see below).

456

457

\section{M. pterygoideus externus pars inferior}

458

459

460

461

462

463

464

465

466

467

468

469

470

471

472

Similarly to the ps-M.p.e., the pi-M.p.e. (Fig. 4B) has a strap-like shape. In contrast with its upper counterpart, the pi-M.p.e. takes its origin on the pterygoid fossa. Specifically, the origin of the pi-M.p.e. is a small flattened area on the lateral surface of the palatine sinus. Its fibers are obliquely oriented and insert dorsally on the neck of the condylar process of the mandible.

\section{M. pterygoideus internus pars anterior}

The M.p.i. is divided into two distinct parts. The pars anterior (pa-M.p.i.; Fig. 4B and 6C) takes its origin on the lateral and ventrolateral surfaces of the palatine sinus. The origin is muscular and spans from the caudal palatine foramen to an area just posterior to the origin of the pi-M.p.e., near the posterior limit of the palatine sinus. The fibers are more oblique anteriorly than posteriorly, and insert on the dorsal part of the pterygoid fossa of the mandibular ascending ramus. The posterior part of the pa-M.p.i. is thinner than the anterior part. The thick portion of the pa-M.p.i. serves as an attachment area for a small anterior projection of the pp-M.mh. (see 'Intermandibular musculature'). 
473

474

475

476

477

478 479 of the small angular process (Fig. 4B).

\section{M. pterygoideus internus pars posterior}

481

482

483

484

485

486

487

488

489

490

491

The pp-M.p.i. (Fig. 4B and 6B,C) consists of a fleshy block that takes its origin on an area located between the posterior part of the palatine sinus and the small fossa anterior to the pterygoid sinus. A coronal section shows that the fibers are obliquely oriented (Fig. S2D). The pp-M.p.i. presents a very small pars reflexa that extends from the anterior- to the posteriormost part of the pterygoid fossa of the ascending ramus, wrapping around the margin

\section{Facial-masticatory musculature}

\section{M. buccinatorius pars externa}

The pe-M.b. (Fig. 6) is a thin sheet of obliquely oriented muscle fibers that envelops the piM.b. and the buccal salivary glands. The muscle takes its narrow and anteroposteriorly elongated origin on the maxilla. Its posterior limit attaches just posteroventral to the zygomatic process of the maxilla. Its anterior part consists of a thin strap on the lateral surface of the maxilla, close to the lateral limit of the nasal cavity. The muscle wraps around the piM.b. and reflects medially to insert along the dorsal part of the lateral surface of the mandible. Its insertion is shorter than its origin, extending from the level of the infraorbital foramen for the posterior two thirds of the length of the horizontal ramus. 
492

493

494

495

496

497

498

499

500

501

502

503

504

505

506

507

508

509

510

511

\section{M. buccinatorius pars interna}

The pi-M.b. (Fig. 6B) is an elongated and fleshy muscle that takes its origin just posterior to the buccal commissure on the ventral part of the lateral surface of the maxilla. The anterior part of the pi-M.b. has a thin projection of its dorsal part that wraps around the lateral border of the dentary pad, to project into the space between the upper and lower jaws. This part of the muscle contacts the salivary glands ventrolaterally. The pi-M.b. lateral surface is enveloped by the pe-M.b. anterior to the zygomatic process of the maxilla. The muscle fibers are horizontally oriented and extend to the level of the caudal nasal foramina. Posteriorly, the pi-M.b. inserts on the dorsal surface of the mandible, at the level of the optic foramen. The insertion is laterally adjacent to that of the pm-M.t.p. It extends anteriorly to reach the level of the maxillary foramen. The orbital part of the pi-M.b. is flattened due to the presence of the large lacrimal gland, dorsally. Madially, it is limited by the presence of the pm-M.t.p.

\section{M. mandibuloauricularis}

The M.ma. (Fig. 6A, C, and D) is a small fleshy muscle with a pseudocylindrical shape. It takes its origin on the anteroventral part of the auricular cartilage. The M.ma. narrows ventrally towards its insertion on a small area of the posterodorsal margin of the angular process of the mandible, between the insertions of the M.m.s. and the M.p.i. The M.ma. presents dorsoventrally directed fibers with a slight medial component. 
512 Intermandibular musculature

513

514 The M.i.a. (Fig. 7; pa-M.mh. sensu Naples, 1999) is a sheet-like muscle that arises from the 515 symphysial cartilage. The M.i.a. fibers are transversely oriented. They insert on both

516 hemimandibles, covering the base of the tongue and the tendon of the geniohyoideus in 517 ventral view (Fig. 7). The M.i.a. is, therefore, a non-bilaterally symmetric muscle (Fig. S2E).

518 It wraps around the ventral margin of the mandible to insert just dorsal to it, on the lateral

519 surface. The M.i.a. extends posteriorly for slightly more than half the length of the horizontal 520

521

522

523

524

525

526

527

528

529

530

531

532

533

\section{M. mylohyoideus pars anterior}

The pa-M.mh. (Fig. 7) is a sheet-like muscle with transversely oriented fibers, and covers the base of the tongue and the long tendon of the geniohyoideus (M.gh., not described). Its morphological similarities with the M.i.a. caused previous studies to describe the latter as a distinct part of the mylohyoideus complex (Naples, 1999). In contrast to the M.i.a., the paM.mh. insertion takes its origin on the ventral part of the medial surface of the mandible, between the widest point of the dentary pad and the pterygoid fossa posteriorly (Fig. 7). In addition to a different insertion, the pa-M.mh. is a bilaterally symmetric element, with both counterparts united medially by a small layer of conjunctive tissue. The pa-M.mh. is slightly thicker than the M.i.a. Posteriorly, the pa-M.mh. anastomoses with the pp-M.mh., the two parts being continuous. In coronal view, the division between the two muscles is characterized by the passage of the sublingual artery (Evans \& De Lahunta, 2013), ventral to the pa-M.p.i. (Fig. S2F). 


\section{M. mylohyoideus pars posterior}

536 The pars posterior of the M. mylohyoideus (pp-M.mh.; Fig. 7) is broader than pa-M.mh.. At

537 the level of the orbital fissure, the sublingual artery (Evans \& De Lahunta, 2013) splits the 538 insertions of the pa-M.mh. and the pp-M.mh.. While the pa-M.mh. inserts on the mandibular 539 ramus, the insertion of the pp-M.mh. extends along the medial surface of the palatine 540 inflation, then along the ventromedial surface of the pterygoid sinus to continue posteriorly to 541 the rostral tympanic process of the petrosal (Fig. 7). Additionally, a thin muscular projection 542 inserts on the medial surface of the pa-M.p.i. Posterior to the hard palate, the pp-M.mh.

543 inserts on the soft palate, keeping its shape until it reaches the anterior part of the 544 stylopharingeus (not described), where it bifurcates. A fleshy fiber extension projects 545 posteriorly to attach on a small area of the anterior surface of the stylohyal, just dorsal to its 546 suture with the epihyal. On the other hand, a ventral sheet-like projection attaches to the 547 tendon of the interstylohyoideus (M.ish., not described; Fig. 7). As in other cases, the tendon 548 could not be segmented. Nevertheless, the presence of muscular fibers of the M.ish. confirm

549 the position of the insertion of the pp-M.mh. described in previous studies (Reiss, 1997). 


\section{Myrmecophaga tridactyla}

\section{Masticatory apparatus}

\section{M. masseter superficialis}

554 In M. tridactyla, the M.m.s. (Fig. 8) is a fleshy and anteroposteriorly elongated muscle. The

555 M.m.s. originates from the ventrolateral margin of the zygomatic process of the maxilla. A 556 strong tendon connects the origin to the almost horizontally oriented muscular fibers. The

557 M.m.s. is thin at the origin, as it overlies the posterior part of the M.m.p. It thickens

558 posteriorly, as it extends anteriorly to the lacrimal foramen and the posterior part of the

559 masseteric fossa. The M.m.s. presents a pars reflexa throughout most of its length (Fig. 8C).

560 The pars reflexa wraps around the ventral edge of the mandible and becomes larger

561 posteriorly, covering only the very posteroventral tip of the small angular process (Fig. 8A,C).

\section{M. masseter profundus}

564 The M.m.p. (Fig. 8B) takes its origin on the anterior part of the ventrolateral surface of the 565 zygomatic arch. Its area of origin includes the small jugal bone and the posteroventral surface 566 of the zygomatic process of the maxilla. The M.m.p. is in contact with the posterior part of the 567 pi-M.b., medially (Fig. 8D). The M.m.p. is obliquely oriented; it inserts ventrally on the 568 mandible and presents a small pars reflexa. The muscle is thick at its origin but thins down 569 posteriorly, where it is overlain by the M.m.s. The M.m.p. is half the length of the M.m.s., 570 with its insertion area stretching from the most posterior part of the masseteric fossa to near 571 the level of the coronoid process. 


\section{M. temporalis superficialis}

574 The M.t.s. (Fig. 8A, C, and D) is a flat muscle covered almost entirely by the large lacrimal

575 gland. It is a fan-like muscle originating from the temporal fossa extending from the level of

576 the optic foramen to the root of the zygomatic process of the squamosal. The lateral surface of

577 the M.t.s. is covered by a tendinous layer that thickens ventrally near the insertion of the

578 muscle on the small coronoid process. While the ventrally converging fibers of the M.t.s.

579 reach the coronoid process posteriorly, the anterior part of the muscle inserts on the mandible

580 uniquely via its tendinous layer (Fig. 8). The M.t.s. is well-separated from the pars

581 zygomatica, posteriorly, due to the very distinct orientation of the muscular fibers.

\section{M. temporalis superficialis pars zygomatica}

584 The pars zygomatica of the M.t.s. (pz-M.t.s.; Fig. 8A, C, and D) is a fleshy and thick part of 585 the M.t.s. complex. It arises from the medial and posteroventral surfaces of the zygomatic process of the squamosal and extends anteroventrally with an oblique orientation. The pzM.t.s. displays a medial portion that extends along the anterior margin of the neck of the mandibular articular process and inserts on the posterior surface of the blunt coronoid process.

589 The lateral part of the pz-M.t.s. is larger and extends along the surface lateral to the mandibular notch. The most ventral part of the pz-M.t.s. is slightly overlain by the dorsal

591 margin of the M.m.s. The pz-M.t.s. is easily distinguishable from its larger counterpart due to 592 the different orientation angle of its fibers. 
594

595

596

597

598

599

600

601

602

603

604

605

606

607

608

609

610

611

612

613

614

615

616

\section{M. temporalis profundus pars lateralis}

The M.t.p. (Figs. 4C and 8B, D) is divided into medial and lateral parts. The pars lateralis (pl-M.t.p.) is a blocky-shaped muscle arising from the ventral limit of the temporal fossa between the anterior tip of the orbital process and the insertion of the ps-M.p.e. The posterior part of the pl-M.t.p. presents a quadrangular shape in lateral view, with the anterior part tapering in near the pi-M.b. The muscular fibers are dorsoventrally oriented with an oblique transversal component. The pl-M.t.p. inserts on the dorsal surface of the ascending ramus deep to the insertion of the M.t.s. While the M.t.p. is well separated from the M.t.s. during the classical dissection, the incomplete staining of the former makes it sometimes hard to delimit. Anteriorly, the insertion of the pl-M.t.p. extends until the level of the anterior margin of the optic foramen (Fig. 8B).

\section{M. temporalis profundus pars medialis}

The pm-M.t.p. (Figs. 4C and 8B, C) in M. tridactyla is a medioventrally extending projection of the pl-M.t.p. Both parts are anastomosed posteriorly, sharing the medial part of the M.t.p. origin. The pm-M.t.p. arises from the ventral surface of the orbital process lateral to the orbital fissure and the foramen rotundum. Slightly anterior to its origin, the pm-M.t.p. extends ventrally on the lateral surface of the ascending ramus (Fig. 4C). Anterior to this point, the two parts of the M.t.p. are distinguished by different insertion areas (Fig. 4C), with pm-M.t.p. reflecting medially. The insertion of the pm-M.t.p. is broad and extends ventrally almost until the level of the mandibular canal. It is limited posteriorly by the mandibular canal. The pmM.t.p. tapers anteriorly to its contact with the posterior part of the pi-M.b. at the orbit midlength. Fiber orientation in the pm-M.t.p. is similar to that of the pl-M.t.p. 


\section{M. pterygoideus externus pars superior}

618 The ps-M.p.e. (Figs. 4C and 8B) is a broad and wide fleshy sheet muscle arising from the 619 large fossa extending from the anteroventral part of the squamosal to the ventral part of the 620 temporal fossa. Its fibers are obliquely oriented and extend posteroventrally to insert on the 621 mandible just anterior to the jaw joint. The posteroventral part of the ps-M.p.e. is

622 characterized by a large pars reflexa that wraps around the medial edge of the articular 623 process. The pars reflexa of the ps-M.p.e. overlays the posterior part of the pars inferior of 624 the M.p.e.

\section{M. pterygoideus externus pars inferior}

627 The pi-M.p.e. (Fig. 4C) is a strap-shaped muscle that originates from the anterior part of the 628 pterygoid fossa, at the level of the optic foramen. In contrast with the ps-M.p.e., the pi-M.p.e. 629 is narrow and elongated. Its origin is thin and lies medial to the pm-M.t.p. The anterior part of 630 the pi-M.p.e. is in tight contact with the pa-M.p.i. The pi-M.p.e. slightly thickens up 631 posteriorly, assuming a circular cross-section. The muscular fibers are horizontally oriented, 632 with an oblique component as they insert posterolaterally on the anterior part of the neck of 633 the articular process (Fig. 4C). The insertion of the pi-M.p.e. reaches about half the length of 634 the neck and is overlain laterally by the pars reflexa of the ps-M.p.e.. The pi-M.p.e. merges 635 with the pars reflexa of the ps-M.p.e. by a thick band of connective tissue. 


\section{M. pterygoideus internus pars anterior}

638 The pars anterior (pa-M.p.i.; Figs. 4C and 8C) is the larger of the two parts of the M.p.i. It 639 takes its origin from the small crest formed by the lateral edge of the palatine. In lateral view, 640 the pa-M.p.i. presents a pseudorectangular shape. Anteriorly, the muscle narrows down (Fig. 641 4C). The most anterior fibers originate just anterior to the level of the ethmoid foramen. The 642 fibers extend ventrally to insert on a lateral prominence of the mandibular ascending ramus, 643 ventral to the passage of the inferior alveolar nerve and artery. Posteriorly, the fibers are 644 dorsoventrally oriented, with an oblique transverse component. Both origin and insertion of 645 the pa-M.p.i. end roughly at the level of the pterygopalatine suture.

The smallest component of the M.p.i. is a fleshy pseudorectangular band in lateral view (Fig. 4C). The origin of the pp-M.p.i. (Figs. 4C and 8C) is very thin and extends from near the

650 palatine-pterygoid suture to the pterygoid sinus at the level of the posterior limit of the jaw 651 joint. The pp-M.p.i. is the continuation of the pa-M.p.i. until the tip of the angular process,

652 where it reaches the insertion area of the M.ma. In lateral view, the fibers are vertically 653 oriented, with a transversal component of about $21^{\circ}$ relative to the sagittal axis of the skull. 654 Posteriorly, the pa-M.p.i. becomes thicker but it tapers off abruptly at the level of the 655 pterygoid sinus. 


\section{Facial-masticatory musculature}

658

659

660

661

662

663

664

665

666

667

668

669

670

671

672

673

674

675

676

677

\section{M. buccinatorius pars externa}

The pe-M.b. (Figs. 8A, C, and D) is an extremely thin sheet enveloping the much thicker pars interna (see below) and the buccal salivary glands. The fibers of the pe-M.b. have an oblique orientation, arising from the long and extremely narrow origin on the maxilla. The origin extends from the level of the most posterior mental foramen to the anterior edge of the zygomatic process of the maxilla. The pe-M.b. extends ventrally, envelopes the pi-M.b. and reflects medially. The muscle wraps around the ventromedial margin of the pars interna of the M. buccinatorius and projects dorsally to insert on the dorsolateral surface of the mandibular horizontal ramus. Its insertion and origin areas are similar in length, but the bad preservation of the soft tissues in the snout did not permit to clearly observe the anterior tip of its origin.

\section{M. buccinatorius pars interna}

The pi-M.b. (Fig. 8B) is extremely long anteroposteriorly, reflecting the elongation of the rostrum. The muscle takes its origin on the maxilla, adjacent to the labial commissure of the mouth, although the muscular fibers arise more posteriorly. The pi-M.b. fibers go on to insert on the dorsal surface of the horizontal ramus of the mandible, ventral to the eye and the lacrimal gland. The fibers have an almost horizontal orientation, leaning slightly ventrally. In cross section, the anterior part of the pi-M.b. is dorsoventrally elongated. The most anterior part of the pi-M.b. presents a medial flap-like projection that rests between both jaws (Fig. 8B). This part of the pi-M.b. contacts the salivary glands laterally. At the length of the 
678 anterior most tip of the nasal, the pi-M.b. drifts ventrally and narrows dorsoventrally (Fig.

679 8C). Posterior to the zygomatic process of the maxilla, the pi-M.b. leans medially to a

680 position between the jaws, deep to the M.m.s. This marks the beginning of the insertion of the

681 pi-M.b., which extends to the anterior part of the insertion of the M.t.p., just anterior to the

682 level of the optic foramen.

683

684

M. mandibuloauricularis

685 The M.m.a. consists of a small fiber bundle that takes its origin from the anterior part of the 686 auricular cartilage. It inserts on the posterior tip of the angular process, between both the pp-

687 M.m.s. and pp-M.p.i. This muscle was damaged on the digitally dissected side of the skull 688 and was described based on its right counterpart.

689

690

Intermandibular musculature

691

\section{M. intermandibularis anterior}

692 The M.i.a. (Fig. 9; pa-M.mh. sensu Naples, 1999a) is extremely elongated, extending for 693 almost half the mandibular length $(127.4 \mathrm{~mm})$. This muscle is very thin and forms a sheet 694 covering the tendon of the genioglossus as well as the tongue (not figured). Each fiber is 695 attached to thin areas on the ventrolateral surfaces of both mandibles. The muscle wraps 696 around the ventral margin of the mandible and stretch transversely to insert on the symmetric 697 element. The fibers are continuous between mandibles. 
700

701

702

703

704

705

706

707

708

709

710

711 Discussion

712

713

714

715

716

717 Myrmecophagidae (Fig. 10; Gibb et al., 2016). Although all anteaters present toothless and

718 elongated jaws, this elongation is particularly pronounced in mymecophagids, reaching 
719 extreme proportions in the giant anteater (M. tridactyla). Pygmy anteaters present a shorter

720 snout, a concave curvature of the basicranial/basifacial axis (Gaudin \& Branham, 1998),

721 pterygoids that do not meet in the midline, as well as relatively well-developed coronoid and

722 angular processes of the mandible (Hirschfeld, 1976; Engelmann, 1985). These, and other

723 morphological traits, are considered ancestral for Vermilingua (Fig. 10; Hirschfeld, 1976;

724 Patterson et al., 1992). Reiss $(1997,2001)$ also found differences between the head

725 musculature of pygmy and myrmecophagid anteaters but overlooked those in the masticatory

726 apparatus.

Our results reveal clear differences in the anatomy of the masticatory muscles of

728

729

730

731

732

733

734

735

736

737

738

739

740 anteaters (Fig. 10). Contrary to myrmecophagids, the pygmy anteater shows a simple $M$.

pterygoideus internus (M.p.i.) without subdivisions, a one-layered masseter (superficialis),

and a relatively larger $M$. temporalis superficialis (M.t.s.) with a bipennate fascicular

architecture (Fig. 10). Additionally, the posterior part of the M. mylohyoideus pars anterior

(pa-M.mh.) inserts on the ventromedial part of the M. pterygoideus internus, unlike in

myrmecophagids (this study; Naples, 1999; Endo et al., 2007, 2017). Lastly, we show the

existence of a two-part M. buccinatorius in the pygmy anteater, contradicting previous

descriptions (Naples, 1985a; Reiss, 1997). These five traits are of potential systematic value

but all were absent in previous comparative studies identifying phylogenetically polarised

muscular traits (Reiss, 1997, 2001).

The subdivision of the M. pterygoideus internus into two parts in myrmecophagids

might be related to size, similar to the increase in the number of facial muscles in anteater species with longer rostra (Naples, 1985a). On the other hand, size differences between 
741 collared and giant anteaters does not affect the M. pterygoideus internus anatomy. The

742 subdivision of this muscle might thus be a diagnostic trait within Vermilingua.

744 in the Northern tamandua and the giant anteater. Our description of a two-unit masseter

745 musculature in myrmecophagids supports the observations made by Endo et al. (2007, 2017),

746 and resembles that of other mammalian groups (e.g., Turnbull, 1970; Naples, 1985b; Endo et

747 al., 1998; Cox \& Jeffery, 2011; Sharp \& Trusler, 2015). A single-unit masseter musculature is

748 therefore an autapomorphy of Cyclopedidae. In the latter taxon, the muscle is attached to the

749 maxilla by a long tendon (Fig. 3A, B). In addition to the lack of an M. masseter profundus

750 (M.m.p.), C. didactylus displays a bipartite M. masseter superficialis (pa-M.m.s. and pp-

751 M.m.s.; Fig. 3A, C, and D), while it is composed of a single block in myrmecophagids (Figs.

752 6A, C, and 8A, C). The pa-M.m.s. in C. didactylus is distinguishable from an M.m.p. because:

753 i) its presents a pars reflexa, typically found in the M.m.s. (e.g., Sharp \& Tusler, 2015); ii) it

754 shares a single tendinous origin with the pp-M.m.s.; iii) a two part M.m.s. with differently

755 orientated muscle fascicles is described in other mammals (e.g., Fig. 3A, Sharp \& Tusler, 756 2015; Wille, 1954).

757 The temporalis complex is also quite distinctive between cyclopedids and

758 myrmecophagids, despite both families presenting deep and superficial muscles (contra Reiss,

759 1997). The temporalis complex is twice as large in cyclopedids compared to myrmecophagids

760 (Table 2). Robust jaw adductor muscles represent an ancestral condition within xenarthrans

761 (Reiss, 2001). Therefore, the presence of large M. temporalis superficialis and profundus in

762 pygmy anteaters is in line with other plesiomorphic musculoskeletal traits previously

763 described (Hirschfeld, 1976; Engelmann, 1985; Reiss, 1997). The bipennate fascicular 
764 arrangement (Fig. S2B) of the M. temporalis superficialis in the pygmy anteater is an

765 ambiguous trait. While it is unique to pygmy anteaters within Vermilingua, fiber pennation is

766 not described in the sloth sister-group (Naples, 1985b). Nevertheless, the loss of bipennate

767 fascicles in the M. temporalis superficialis might be an autapomorphic trait of

768 myrmecophagids, given that other mammals present either bipennate or multipennate fiber

769 arrangements (Woods \& Howland, 1979; Taylor \& Vinyard, 2009; Hautier, 2010). Curiously,

770 the pars zygomatica of the M. temporalis superficialis is relatively smaller in C. didactylus

771 than in myrmecophagids (Table 2), suggesting that the posterior component of force of the

772 temporalis complex is less important in pygmy anteaters.

773

In addition, to the differences listed above, we recognize, for the first time, the

774

775

776

777

778

779

780

781

782

783

784

785

786

presence of an individualized M. intermandibularis anterior (M.i.a.) in the Vermilingua (Figs. 5B, 7B, and 9B). Naples (1999) considered this muscle to be a part of the M. mylohyoideus (M.mh.). We show that this muscle is attached to the ventrolateral margin of the anterior part of the lower jaws (Figs. 5B, 7B, and 9B), which contrasts with the insertion area of the M.mh. Furthermore, we confirm that the M.i.a. is made of transversally continuous fibers. The paM.mh. and pp-M.mh. comprise two bilaterally symmetric muscles that join along a midline axis (Fig. S2C). A similar condition is found in sloths (Naples, 1986), as well as in other mammals like moonrats (Turnbull, 1970), nectarivorous bats (Wille, 1954), and humans (Gray, 1995). Turnbull (1970) uses two criteria to assign a M. digastricus pars anterior to the M.mh.: i) the presence of intertonguing connection at the midline, and ii) the contiguity of the attachment on the mandible. None of these conditions were found in the anteater "pa-M.mh." (sensu Naples, 1999). Therefore, we propose to consider this muscle as the M.i.a. (Diogo et al., 2008). The pa-M.mh. (sensu Naples, 1999), the M. transversus mandibularis of rats 
787 (Greene, 1935), and the pa-M.mh. of tree-shrews (Le Gros Clark, 1924) are developmentally

788 distinct from the M.mh. (Diogo et al., 2008). The muscle referred to by Le Gros Clark (1924),

789 Greene (1935), and Naples (1999) is developmentally homologous with the sarcopterygian

790 M.i.a. while the M.mh. is homologous to the M. intermandibularis posterior (Diogo et al.,

791 2008). This muscle is mostly present in mammals with highly mobile mandibular symphysis,

792 serving as a stabilizer (Hiiemae \& Houston, 1971).

793

794

795

796

797

798

799

800

801

802

803

804

805

806

807

808

Overall, the results of our detailed descriptions and comparisons of the masticatory apparatus of anteaters provide several morphological traits that can be useful for systematics purposes. The previously unaccounted differences between the masticatory muscles of cyclopedids and myrmecophagids emphasize the level of morphological divergence acquired during the evolution of this clade with a highly specialized diet. We highlight the importance of soft-tissues as a source of diagnostic traits by combining conventional dissection with diceCT (Metscher, 2009). Our results allow us to propose that a two part masseter musculature associated with a jugal bone and an unfused mandibular symphysis presenting an $M$. intermandibularis anterior are the plesiomorphic condition for Vermilingua. On the other hand, plesiomorphic architecture and relative size of the temporalis complex are impossible to predict, as these differ between extant sloth genera (Naples, 1985b) and data for armadillos (their xenarthran outgroup) are scarce and inconclusive (Kuhlhorn, 1939 in Turnbull, 1970).

\section{Mandibular mechanics}

Regardless of the numerous differences discussed in the previous section, the masticatory apparatus of anteaters can be generally characterized by a set of adaptations to 
809 myrmecophagy like the complete tooth loss, the loss of masticatory capabilities (Naples,

810 1999), the reduction of masticatory muscles (Reiss, 1997; Naples, 1999; Endo et al., 2007,

811 2017), and the unfused mandibular symphysis (e.g., Ferreira-Cardoso, Delsuc \& Hautier,

812 2019). The loss of chewing ability is well illustrated by the absence of the main mandibular

813 abductor, the digastricus muscle (e.g., Turnbull, 1970; Hylander, Johnson \& Crompton, 1987;

814 Hylander, 2006) in all dissected specimens.

The loss of a typical mandibular adduction/abduction cycle evolved with a new

816 feeding strategy involving protrusion-retraction movements of an elongated sticky tongue

817 (Montgomery, 1983, 1985). Naples (1999) associated this type of movement with the unfused

818 mandibular symphysis in the giant anteater. The proposed model suggests that the loose

819 symphysis allows for hemimandibular roll in order to increase the volume in the oral cavity

820 (mouth opening) during tongue protrusion (Naples, 1999; Figs. 11A-C). The medial roll of the

821 dorsal margin of the mandibular body (mouth opening; Fig. 11C top) is achieved by the

822 contractions of the masseter and $M$. temporalis superficialis muscles. The former contributes

823 to the lateral roll of the angular process of the mandible, while the latter contributes to the

824 medial roll of the coronoid process and additionally performs retraction movements (Figs.

825 11A, B; Naples, 1999). Mouth closing (Fig. 11C, F bottom) results from the lateral roll of the

826 dorsal edge of the mandible, which is achieved by the contraction of the M. pterygoideus

827 internus (Fig. 11A, B; Naples, 1999). The M. temporalis profundus also contributes to

828 mandibular closing by medially rolling the ascending ramus (Fig. 11 B; Naples, 1999). The

829 contraction of the well-developed M. intermandibularis anterior (M. mylohyoideus pars

830 anterior sensu Naples, 1999) additionally contributes to hemimandibular roll (Naples, 1999).

831 This contraction medially rotates the ventral margin of the mandibular rami, causing the 
832 lateral roll (Fig. 11C down) of their dorsal edges (mouth closing; Naples, 1999). Collared

833 anteaters probably show similar mandibular mechanics as they show many anatomical

834 similarities with giant anteaters (Fig. 11A-C; Endo et al., 2017).

A biomechanical model of the masticatory apparatus of pygmy anteaters is yet to be

836 proposed. On the one hand, cyclopedids and myrmecophagids present several muscular and

837 osteological differences (see previous section of 'Discussion'). On the other hand, key

838 similarities such as a large M. intermandibularis anterior, a reduced masseter, and an unfused

839 mandibular symphysis suggest that both families share the same roll-dominated

840 hemimandibular movements. Additionally, all anteaters present a typical mandibular

841 innervation pattern composed of dorsal canaliculi that were putatively associated to the

842 coordination between hemimandibular rolling and tongue protrusion in anteaters (Ferreira-

843 Cardoso, Delsuc \& Hautier, 2019).

844

We propose that food ingestion in pygmy anteaters happens through hemimandibular

845 roll similar to that in myrmecophagids. However, the large coronoid process/temporalis

846 musculature in pygmy anteaters suggest a relatively higher bite force magnitude (Jones, 1997;

847 Jaskolka, Eppley \& van Aalst, 2007; Nogueira, Peracchi \& Monteiro, 2009). The evolution of

848 a large temporalis complex is associated with an increase in crushing force (e.g., Jones,

849 1997). Pygmy anteaters toothlessness and associated myrmecophagous diet suggest that

850 relative muscular volumes are insufficient to characterize the masticatory mechanics, and that

851 different mandibular mechanics may result from similar muscle proportions. The bipennation

852 of the pygmy anteater M. temporalis superficialis indicates that this muscle is likely

853 responsible for a majority of the force applied during mandibular movement (see 'Muscle- 
854 bone interactions'; Avis, 1959; Amorim et al., 2008). Therefore, the mediolateral roll is likely 855 temporalis-led in pygmy anteaters.

856 We argue that the lateral orientation of the coronoid process (Figs. 3B, 4A, 11D, E)

857 and the M. temporalis superficialis medial line of action in the pygmy anteater (Fig. 11E) are

858 the basis for the temporalis-led medial roll of the dorsal margin of the mandibular body

859 (mouth opening; Fig. 11F top). This contrasts with mouth opening in myrmecophagids, in

860 which the lateral roll of the angular process is putatively led by the M. masseter superficialis

861 (larger relative contribution; Table 2). A similar temporalis-led hemimandibular roll is found

862 in the tailless tenrec (Tenrec ecaudatus; Oron \& Crompton, 1985), which presents a highly

863 mobile mandibular symphysis (Mills, 1966) and lacks a M. masseter profundus (Oron \&

864 Crompton, 1985). These traits grant a high mediolateral mobility during mandibular

865 adduction (Oron \& Crompton, 1985). Interestingly, masseter/temporalis relative proportions

866 in the tailless tenrec (Turnbull, 1970) are similar to those of the pygmy anteater, and so is the

867 lateral orientation of their coronoid process (e.g., Fig. 11D, E). The absence of a M. masseter

868 profundus implies that the force vector of the masseter complex has a much reduced vertical

869 component compared to other mammals (Weijs, 1980; Gueldre \& de Vree, 1990; Cox et al.,

870 2012). In addition, we propose that the contraction of the transverse fibers of the $M$.

871 mylohyoideus pars anterior (M. mylohyoideus pars media sensu Naples, 1999) also

872 contributes to the medial roll of the dorsal margin of the ascending ramus (Fig. 11E), by

873 applying a ventromedial force (Figs. 5C, 7C, and 9C). The contraction of the M. pterygoideus

874 internus, M. temporalis profundus pars medialis, and M. intermandibularis anterior applies

875 the medial force on the ventral margin of the mandibular ramus (Fig. 11E) during mouth

876 closing (Fig. 11F down). In opposition to myrmecophagids, the pars reflexa of the $M$. 
877 masseter superficialis in the pygmy anteater is relatively well-developed and wraps around

878 the full length of the ventral margin of the ascending ramus (Fig. S2A). This might indicate

879 that the M. masseter superficialis retains an elevator function (Hylander, 2006), and that it

880 might facilitate the mouth closing, by adducting the mandibular rami (e.g., Hiiemae, 1971).

881 In sum, cyclopedids and myrmecophagids evolved similar mandibular movements

882 (roll-dominated) despite the evolution of divergent skull shapes and sizes (Reeve, 1940). This

883 functional adaptation of mandibular dynamics to tongue protrusion/retraction cycles in

884 Vermilingua could represent a new example of many-to-one-mapping of form to function

885 (Wainwright et al., 2005; Strobbe et al., 2009). Future functional comparisons between

886 pygmy and myrmecophagid anteaters should include estimations of mechanical and bite force 887 potential (e.g., Cornette et al., 2012), and a better characterization of the functional output of

888 the jaw system using biomechanical models (e.g., Cleuren, Aeris \& De Vree, 1995).

889

890

\section{Muscle-bone interactions}

891

In the previous sections we discussed the differences between the masseter and temporalis

muscle complexes between cyclopedids and myrmecophagids. The osteological divergence

893

between these families partly reflects the temporalis/masseter trade-off that is key to

894

understand the similar functional output of their masticatory apparatus. While the masseter

895

musculature is reduced in cyclopedids, the M. temporalis superficialis is especially enlarged

896

when compared to myrmecophagids (Table 2). These muscles' origin and insertion areas

897 correspond to major osteological differences between pygmy and myrmecophagid anteaters

898 (e.g., Reiss, 1997; Ferreira-Cardoso, Delsuc \& Hautier, 2019). On the one hand, pygmy 
899 anteaters lack a jugal bone, which is the origin of the M. masseter profundus in

900 myrmecophagids (this study; Naples, 1999; Endo et al., 2017) and other mammals (Turnbull,

901 1970; Cox \& Jeffery, 2011; Fabre et al., 2017; Ginot, Claude \& Hautier, 2018). On the other

902 hand, the main surface for the insertion of the M. temporalis superficialis - the coronoid

903 process - has almost vanished during the evolution of myrmecophagids. This observed

904 covariation pattern between muscles and bones can be partly explained by the muscle-bone

905 interactions that occur during embryonic development (Cheverud, 1982; Hallgrímsson et al.,

906 2007; Zelditch et al., 2008). The absence/reduction of muscular contraction results in skeletal

907 anomalies, including the loss or reduction of certain bones and cartilages (Hall \& Herring,

908 1990; Atchley \& Hall, 1991; Rot-Nikcevic et al., 2006).

The loss of the jugal bone in pygmy anteaters could be linked to the absence of a $M$.

910 masseter profundus (M.m.p.). Similar conditions are present in the tailless tenrec (Tenrec

911 ecaudatus; Oron \& Crompton, 1985) and the Asian house shrew (Suncus murinus;

912 (Fearnhead, Shute \& Bellairs, 1955). However, the absence of a jugal bone does not always

913 imply the loss of the M.m.p. (e.g., Crocidura russula; Cornette, Tresset \& Herrel, 2015). Such

914 cases may result from either early bone fusion (e.g., jugal + maxilla; Tavares et al., 2017), or

915 the inactivation of genes that induce bone development (e.g., Kist, Greally \& Peters, 2007).

916 Additionally, the M.m.p. could either not differentiate during ontogeny or secondarily fuse

917 with the M. masseter superficialis, as reported for other muscle complexes (e.g., Diogo,

918 2018). Most mammals (e.g., Turnbull, 1970; Sharp \& Trusler, 2015), including sloths

919 (Naples, 1985b) and myrmecophagid anteaters, present jugal bones and an M.m.p. Therefore,

920 the complete loss of a functional zygomatic arch and the M.m.p. in the pygmy anteater offers 
921 a striking example of developmental integration linked to muscle-bone interaction, as well as

922 an empirical evidence of modularity within the masticatory apparatus.

923 The reduction of both the coronoid process of the mandible and the M. temporalis

924 superficialis in myrmecophagid anteaters also represents a classical example of structural

925 covariance. Although the initiation of the coronoid development is an intrinsic process to the

926 mandibular ossification, its growth is dependent on mechanical loading applied by the

927 temporalis musculature (Avis, 1959; Amorim et al., 2008; Anthwal, Peters \& Tucker, 2015).

928 Anthwal and colleagues (2015) showed that reduced temporalis musculature correlated with

929 weakly-developed coronoid processes in mice. The contrast between the large $M$. temporalis

930 superficialis $(42.2 \%)$ and prominent coronoid process in pygmy anteaters, and the much

931 smaller muscle $(13.5 \%-20.9 \%)$ and almost nonexistent process in myrmecophagids is a good

932 example of muscle-induced coronoid development in non-model organisms. In addition to

933 size, bipennate muscles in pygmy anteaters generate relatively larger forces (increased

934 physiological cross-section areas) than unipennate ones in myrmecophagids (Turnbull, 1970;

935 Gans \& de Vree, 1987; Hylander, 2006), which further indicates a decrease of medially

936 rotating forces applied on the coronoid process in the myrmecophagids.

937 While the examples discussed above are a good illustration of contrasting

938 morphofunctional patterns between cyclopedids and myrmecophagids, the loss of the

939 zygomatic arch represents a common developmental trend. All anteaters putatively lack a

940 zygomaticomandibularis muscle (Edgeworth, 1923; Naples, 1999). This muscle originates

941 from the zygomatic arch in sloths (Naples, 1985b) and other mammals (Turnbull, 1970; Cox

942 \& Jeffery, 2011; Sharp \& Trusler, 2015). Although the absence of muscular contraction by

943 the zygomaticomandibularis muscle could provide a developmental explanation for the loss of 
944 the zygomatic arch (e.g., Hall \& Herring, 1990; Herring, 1993), we argue that the

945 zygomaticomandibularis was not completely lost in anteaters. We found a pars zygomatica of

946 the M. temporalis superficialis in the three species of anteaters, especially well-separated in

947 the pygmy anteater. Naples (1999) homologized this muscle in M. tridactyla with a

948 homonymous structure in the two-toed sloth (Choloepus sp.). However, the pars zygomatica

949 of the M. temporalis superficialis of anteaters inserts along the lateral part of the mandibular

950 notch (Figs. 1B, 3, 6, 8), instead of the anterior edge of the coronoid process as in the two-

951 toed sloth (1985b). Therefore, we dispute the homology implied by Naples (1999), and

952 propose that the pars zygomatica of the M. temporalis superficialis of anteaters might

953 correspond to a zygomaticomandibularis pars posterior. The origin and insertion of this

954 muscle is similar to the zygomaticomandibularis pars posterior described in rodents (e.g.,

955 Cox \& Jeffery, 2011; Fabre et al., 2017; Ginot, Claude \& Hautier, 2018). Previous

956 descriptions of this muscle as a part of the M. temporalis superficialis (Naples, 1985b, 1999)

957 can be justified by the common developmental origin of the two muscles (e.g., Edgeworth,

958 1914). Previous studies described the zygomaticomandibularis as not separable from either

959 the masseter or the temporalis complexes in some carnivores, ungulates, bats, and marsupials

960 (Druzinsky, Doherty \& De Vree, 2011 and references therein). Nevertheless, we propose that

961 the designation of pars zygomatica of the M. temporalis superficialis should be used until

962 further embryological evidence is available in anteaters. 


\section{Dietary versus functional convergence}

965 Myrmecophagy is a textbook example of evolutionary convergence linked to dietary

966 adaptation (McGhee, 2011). However, a comprehensive comparative study of the masticatory

967 apparatus of all ant- and termite-eating placental lineages is yet to be undertaken. Reiss

968 (2001) took a first step in this direction, but this study included pangolins, anteaters, and

969 respective sister taxa only. Furthermore, Reiss (2001) focused on the systematic implications

970 of convergence (i.e., homoplasy), with morphofunctional considerations focusing mostly on

971 tongue musculature.

972 Pangolins are a well-known example of ecological and morphological convergence

973 with anteaters (Rose et al., 2005; McGhee, 2011). While several studies have been dedicated

974 to the head musculature of pangolins (e.g., Macalister, 1875; Windle \& Parsons, 1899;

975 Edgeworth, 1923; Imai, 1978; Endo et al., 1998), quantitative elements (volume or mass

976 ratios) and functional interpretations are almost nonexistent. Despite this lack of information,

977 both groups present evident muscular differences. First, the masseter complex appears to be

978 more complex than in anteaters, with three parts described in Manis javanica (Sunda

979 pangolin; Endo et al., 1998; Fig. 7). Windle and Parsons (1899) reported that the masseter

980 takes its origin on a "fibrous zygoma". Differences in fiber orientation are not provided (Endo

981 et al., 1998), although the most anterior bundle appears to be the most vertically oriented (Fig.

982 7; Endo et al., 1998). This might suggest the existence of a M. masseter superficialis with two

983 layers, as in C. didactylus, with a small M. masseter profundus (M.m.p.) anteriorly. However,

984 this cannot be confirmed based on the existing bibliography. Edgeworth (1923) describes the

985 M. masseter as arising from the "lower margin of the zygomatic portion of the superior

986 maxilla", while a more oblique muscle arises from the medial surface of the zygomatic arch 
987 (“M. zygomaticomandibularis”, M.zm.). The figures associated with Edgeworth's (1923; Figs.

98859 and 60) study suggest that the described M.zm. could also correspond to an M.m.p. (Table

989 4). Considering the available information, establishing clear homologies with the masseter

990 complex of anteaters is not possible. Nonetheless, if pangolins have a "true" M.zm., this

991 muscle is not homologous to a putative M.zm. in anteaters, given the much more posterior

992 origin and insertion of the latter (see 'Muscle-bone interactions').

The pterygoideus complex of pangolins is composed of a M. pterygoideus externus

994 (M.p.e.), and a M. pterygotympanicus (absent in anteaters; Edgeworth, 1923; Endo et al.,

995 1998). Edgeworth (1923) described a M. pterygoideus internus (M.p.i.) that is atrophied during development, while Yeh (1984) and Endo et al. (1998) reported its absence in adult pangolins. As for the M.p.e., Endo et al. (1998) suggested the presence of two muscle bundles, probably corresponding to the pi-M.p.e. and the ps-M.p.e. of anteaters and other mammals (e.g., Turnbull, 1970). While the ps-M.p.e. arises from the parietal bone in anteaters, both parts arise from the pterygoid in pangolins (Endo et al., 1998).

Parsons, 1899; Edgeworth, 1923; Endo et al., 1998; Table 4). It is, nevertheless, less developed than in other mammals (e.g., Turnbull, 1970). been described (Edgeworth, 1923). However, both present a medial raphe, which suggests that these elements are homologous to the M. mylohyoideus pars anterior (pa-M.mh.) and posterior (pa-M.mh.) of anteaters (Table 4). The attachment of the latter to the pterygoid bone (Edgeworth, 1923) is similar to the condition in T. tetradactyla (Fig. 7). Endo et al. (1998) described a single M.mh. in M. javanica, but no M.i.a. The available information suggests the 
1010 absence of a true M.i.a. (sensu Diogo et al., 2008) in pangolins, which is congruent with the 1011 presence of a fused symphysis.

1012

The divergence between the musculature of anteaters and pangolins reflects the

1013 distinct evolutionary histories of these two placental groups (Meredith et al., 2011).

1014 Furthermore, key aspects of the pangolin masticatory apparatus like the absence of an $M$.

1015

1016

1017

1018

1019

1020

1021

1022

1023

1024

1025

1026

1027

1028

1029

1030

1031

1032 intermandibularis anterior, the presence of an M. digastricus, and the fused mandibular

symphysis suggest the evolution of completely different mechanics from the hemimandibular

roll in anteaters. Different nomenclatures, heterogeneous levels of details, and limited

illustrations in previous studies make a unifying study of the head musculature of pangolins a prerequisite for further interpretations.

The aardvark (Orycteropus afer) head musculature is substantially better described than that of pangolins. The head muscles of the aardvark have been the subject of several studies (Humphry, 1868; Galton, 1869; Windle \& Parsons, 1899; Bender, 1909; Edgeworth, 1924; Sonntag, 1925; Frick, 1951). Similar to other myrmecophagous species, the aardvark also possesses an elongated and specialized tongue (Goździewska-Harłajczuk, KlećkowskaNawrot \& Barszcz, 2018), an elongated snout, and a regressed dentition lacking enamel.

However, the aardvark presents a developed ascending ramus of the mandible, with prominent coronoid and condylar processes, a broad masseteric fossa (e.g., Edgeworth, 1924; Ferreira-Cardoso, Delsuc \& Hautier, 2019), and is able to chew (Patterson, 1975).

The masseter complex of $O$. afer is composed of multilayered M. masseter superficialis (M.m.s.) and profundus (M.m.p.; Frick, 1951). Similar to anteaters, the M.m.s. is the largest (Frick, 1951). According to Frick (1951) it is divided into three layers, the anterior two originating from the zygomatic process, while the most posterior originates from the

Peer] reviewing PDF | (2020:03:46708:1:1:NEW 26 Jun 2020) 
1033 jugal. The M.m.s. presents a large pars reflexa posterodorsally (Edgeworth, 1924; Sonntag, 1034 1925; Frick, 1951). The M.m.p. of O. afer is divided into two sublayers (Frick, 1951). The 1035 architecture of the masseter complex of $O$. afer appears to be more complex than that of both 1036 anteaters and pangolins. Unlike anteaters, O. afer presents a M. zygomaticomandibularis (M.zm.) that inserts

1038

1039

1040

1041

1042

1043

1044

1045

1046

1047

1048

1049

1050

1051

1052

1053

1054

1055 on the lateral surface of the mandible dorsally to the masseter complex. This muscle has vertically oriented fibers and originates along the posteroventral part of the jugal and the anterior part of the zygomatic process of the squamosal (Edgeworth, 1924; Sonntag, 1925;

Frick, 1951). It is probably not related to the M. temporalis superficialis pars zygomatica of anteaters, as its insertion on the mandible is much more anteroventral and its origin further apart from the temporal fossa (Edgeworth, 1924).

The temporalis complex of $O$. afer differs from that of anteaters as it extends dorsally and posteriorly into the cranial vault and fully covers the coronoid process (Edgeworth, 1924; Sonntag, 1925). This complex is divided into three parts (Frick, 1951). The posterior part is the largest and might be homologous to the M. temporalis superficialis (M.t.s.) and its pars zygomatica (pz-M.t.s.) in anteaters (Table 4). In contrast to anteaters, the origin of this muscle stretches posterodorsally into the parietal (Edgeworth, 1924; Sonntag, 1925; Frick, 1951). The smaller medial part of the masseter complex of $O$. afer appears to be absent in anteaters.

Firstly, fiber separation of a medial portion of the M.t.s. was absent in vermilinguans.

Secondly, the medial part of the M.t.s. of $O$. afer arises from the most anterior part of the parietal and the posterior part of the postorbital process (Frick, 1951), which is not developed in anteaters. The deepest part of the temporalis of $O$. afer (Frick, 1951) corresponds to the $M$. temporalis profundus of anteaters (Table 4).

Peer) reviewing PDF | (2020:03:46708:1:1:NEW 26 Jun 2020) 
1057

1058

1059

1060

1061

1062

1063

1064

1065

1066

1067

1068

1069

1070

1071

1072

1073

1074

1075

1076

1077

1078

of anteaters (Edgeworth, 1924; Sonntag, 1925; Frick, 1951). Its M. pterygoideus internus

presents three parts, while only two were identified in M. tridactyla and T. tetradactyla, and a single one in C. didactylus. The M. pterygoideus externus is similar to that of anteaters, being separated into superior and inferior heads arising from the alisphenoid (Frick, 1951), although neither Edgeworth (1924) nor Sonntag (1925) refer to such division. As in pangolins, the aardvark presents a pterygotympanicus that originates on the ectotympanic and displays a tendinous connection to the tensor veli palatini, on the palate (Edgeworth, 1924; Sonntag, 1925).

Edgeworth (1924) described a longitudinally oriented "intermandibularis" with bifid tendinous insertions on the ventrolateral surface of the mandibles of $O$. afer. Sonntag (1925) suggested that this muscle might correspond to the M. mylohyoideus (M.mh.). We propose that the aardvark's "intermandibularis" is homologous to the M. geniohyoideus (M.gh.) of anteaters (Table 4), as both have longitudinally oriented fibres, present an anterior bifurcation of the muscular fibers, and take their origin on the ceratohyal and basihyal (Edgeworth, 1924; Sonntage, 1925; Frick, 1951). Such traits are present in other mammals such as sloths (Naples, 1986), dogs (Evans \& De Lahunta, 2013; e.g., Fig. 6-22), and humans (Drake et al., 2015). This homology appears to agree with Frick (1951), despite this author's introduction of a new term, "intermandibularis profundus", and contradictory illustration showing the bifurcate tendon associated with the M.mh. (Abb. 8; Frick, 1951). Edgeworth (1924) and Sonntag (1925) suggested that a transversely oriented muscle attaching medially to the posterior half of the aardvark mandible to be a "M. digastricus anterior". This muscle presents a variable median raphe (Edgeworth, 1924; Frick, 1951), such as the mylohyoideus

Peer] reviewing PDF | (2020:03:46708:1:1:NEW 26 Jun 2020) 
1079 complex in anteaters and other mammals (Edgeworth, 1914; Saban, 1968; Turnbull, 1970),

1080 and it does not connect to the M. digastricus pars posterior (Edgeworth, 1924; Sonntag,

1081 1925). Therefore, we agree with Frick’s (1951) interpretation that the “M. digastricus

1082 anterior" (sensu Edgeworth, 1924 and Sonntag, 1925) of the aardvark is homologous to the

1083 M.mh. (Table 4). It probably corresponds to the M. mylohyoideus pars anterior of anteaters,

1084 given its insertion area. The M. mylohyoideus pars posterior is also possibly present in $O$. afer

1085 Frick (1951), but this remains to be confirmed.

1086

In O. afer, both pars anterior and posterior of the M. digastricus (M.di.) appear to be

1087

present (Humphry, 1868; Frick, 1951), while both are absent in anteaters. The presence of the

M.di. suggests that mandibular depression in O. afer happens as in other mammals (e.g.,

1089

Turnbull, 1970; Hylander, 1987), in contrast with anteaters.

1090

In sum, the aardvark masticatory musculature is much more similar to those of non-

1091

myrmecophagous placentals than to that of anteaters. This is not surprising, given the level of

1092

divergence between these two myrmecophagous lineages (Meredith et al., 2011), their

1093

different states of tooth reduction (Meredith et al., 2009), and their obvious differences in

1094

skull morphology (Davit-Béal, Tucker \& Sire, 2009). Furthermore, the articular condyle is

1095

well dorsal to the tooth row in aardvarks, which generally increases the momentum of force

1096

applied to food items (Greaves, 2012). Despite being myrmecophagous, the aardvark actively

1097

chews in order to obtain water from cucumber-like plants (Patterson, 1975). The large

1098

temporalis and masseter muscles (Edgeworth, 1924; Sonntag, 1925; Frick, 1951), the

1099

presence of an ossified zygomatic arch, and the fused mandibular symphysis imply significant

1100

functional differences from the anteater hemimandibular roll model. The masticatory

1101 apparatus in aardvarks does not appear to have undergone a morphofunctional shift during the 
1102 evolution of myrmecophagy. Thus, aardvarks, pangolins, and anteaters constitute three

1103 examples of convergent dietary specialization with different mastication mechanics. This

1104 suggests that the plasticity of the mammalian masticatory apparatus played a key role in the

1105 convergent evolution of rapid ingestion of small-sized food items in myologically divergent

1106 lineages.

1107

1108 Conclusion

1109 Here we describe the masticatory, facial-masticatory, and intermandibular muscles of the

1110 three extant anteater genera. While collared and giant anteaters show very similar

1111 morphologies, the masticatory apparatus of the pygmy anteater exhibits marked differences.

1112 These include important discrete morphological traits with systematic value. We also propose

1113 that muscle-bone interactions play a major role in the morphological and functional

1114 differentiation between the two anteater lineages. Our proposed mastication model for the

1115 pygmy anteater suggests that tongue protrusion-retraction movements co-evolved with

1116 hemimandibular roll in both cyclopedids and myrmecophagids. This provides a fine example

1117 of many-to-one mapping (Wainwright et al., 2005; Strobbe et al., 2009), disagreeing with

1118 previous interpretations of similarity between the masticatory apparatus of the two anteater

1119 families (Reiss, 1997). Further comparison with available data from the literature show that

1120 the biomechanics of anteaters may well differ from that of pangolins despite their ecological

1121 convergence (McGhee, 2011). Further studies will be needed to assess biomechanical

1122 differences, notably the magnitude of forces applied to the hemimandibular roll (e.g., Oron \&

1123 Crompton, 1985), and to precisely characterize mandibular movement in anteaters and 
1124 pangolins and further explore the biomechanics of their masticatory apparatus in the context

1125 of convergent evolution towards myrmecophagy.

1126

1127 Acknowledgements

1128 We thank the three anonymous reviewers for their comments and suggestions. We

1129 acknowledge the JAGUARS collection from the INDIGEN project, and François Catzeflis

1130 (Institut des Sciences de l'Evolution de Montpellier) for providing access to the specimens

1131 used in this study. We would also like to thank Anthony Herrel and Kévin Le Verger for their

1132 help (Muséum National d'Histoire Naturelle de Paris). We thank Renaud Lebrun for his

1133 assistance with the $\mu$-CT scanner, as well as the MRI platform. We would additionally like to

1134 thank Mélanie Debiais-Thibaud (Institut des Sciences de l'Evolution de Montpellier) and

1135 Samuel Ginot (Institut de Génomique Fonctionnelle de Lyon) for discussions about staining

1136 protocols, and Marie-Ka Tilak (Institut des Sciences de 1'Evolution de Montpellier) for her

1137 help in the molecular biology laboratory. This is contribution ISEM 2020-xxx of the Institut

1138 des Sciences de 1'Evolution de Montpellier.

1139 References

1140 Alroy J. 1999. The fossil record of North American mammals: evidence for a Paleocene

1141 evolutionary radiation. Systematic Biology 48:107-118. DOI:

1142 $10.1080 / 106351599260472$. 
1143 Amorim MM, Borini CB, Lopes SLP de C, Haiter-Neto F, Bérzin F, Caria PHF. 2008.

1144 Relationship between the inclination of the coronoid process of the mandible and the

1145 electromyographic activity of the temporal muscle in skeletal Class I and II individuals.

1146 Journal of Oral Science 50:293-299. DOI: 10.2334/josnusd.50.293.

1147 Anthwal N, Peters H, Tucker AS. 2015. Species-specific modifications of mandible shape reveal independent mechanisms for growth and initiation of the coronoid. EvoDevo 6:35. DOI: $10.1186 / \mathrm{s} 13227-015-0030-6$.

1150

1151

1152

1153

1154

1155

1156

1157

1158

1159

1160

1161

1162

1163

Atchley W, Hall B. 1991. A model for development and evolution of complex morphological structures. Biological Reviews 66:101-157. DOI: 10.1111/j.1469-185X.1991.tb01138.x.

Avis V. 1959. The relation of the temporal muscle to the form of the coronoid process. American Journal of Physical Anthropology 17:99-104. DOI: 10.1002/ajpa.1330170204.

Barlow KE, Jones G, Barratt EM. 1997. Can skull morphology be used to predict ecological relationships between bat species? A test using two cryptic species of pipistrelle. Proceedings of the Royal Society of London. Series B: Biological Sciences 264:16951700. DOI: $10.1098 / \mathrm{rspb} .1997 .0235$.

Bender O. 1909. Die Halsorgane von Orycteropus afer= capensis (Pallas) und Tamandua tetradactyla L. Denkschriften Der Medicinisch-Naturwissenschaftlichen Gesellschaft zu Jena 15:373-400.

Cheverud JM. 1982. Phenotypic, genetic, and environmental morphological integration in the cranium. Evolution 36:499-516. DOI: 10.1111/j.1558-5646.1982.tb05070.x.

Cleuren J, Aeris P, De Vree F. 1995. Bite and joint force analysis in Caiman crocodilus. 
1165 Cornette R, Herrel A, Cosson J-F, Poitevin F, Baylac M. 2012. Rapid morpho-functional 1166 changes among insular populations of the greater white-toothed shrew. Biological 1167 Journal of the Linnean Society 107:322-331. DOI: 10.1111/j.1095-8312.2012.01934.x.

1168 Cornette R, Tresset A, Herrel A. 2015. The shrew tamed by Wolff's law: do functional 1169 constraints shape the skull through muscle and bone covariation? Journal of Morphology $1170 \quad$ 276:301-309. DOI: 10.1002/jmor.20339.

1171 Cox PG, Jeffery N. 2011. Reviewing the morphology of the jaw-closing musculature in 1172 squirrels, rats, and guinea pigs with contrast-enhanced microCT. The Anatomical Record 1173 294:915-928. DOI: 10.1002/ar.21381.

1174

Cox P, Jeffery N. 2015. The muscles of mastication in rodents and the function of the medial pterygoid. In: Cox P, Hautier L eds. Evolution of the rodents: advances in phylogeny, functional morphology and development. Cambridge University Press ..., 350-372.

Cox PG, Rayfield EJ, Fagan MJ, Herrel A, Pataky TC, Jeffery N. 2012. Functional evolution 1178 of the feeding system in rodents. PLoS ONE 7. DOI: 10.1371/journal.pone.0036299.

Cuvier G. 1798. Tableau élémentaire de l'histoire naturelle des animaux. Paris: Baudouin.

1180

1181

1182

1183

1184

Davit-Béal T, Tucker A, Sire J. 2009. Loss of teeth and enamel in tetrapods: fossil record, genetic data and morphological adaptations. Journal of anatomy 214:477-501. DOI: doi.org/10.1111/j.1469-7580.2009.01060.x.

Diogo R. 2018. Head and neck muscle evolution from sarcopterygian fishes to tetrapods, with a special focus on mammals. In: Diogo R, Ziermann J, Molnar J, Siomava N, Abdala V 

eds. Muscles of chordates: development, homologies, and evolution. Boca Raton, Florida: CRC Press,.

1187

1188

1189

1190

1191

1192

1193

1194

1195

1196

1197

1198

1199

1200

1201

1202

1203

1204

1205

Diogo R, Abdala V, Lonergan N, Wood BA. 2008. From fish to modern humans comparative anatomy, homologies and evolution of the head and neck musculature. Journal of Anatomy 213:391-424. DOI: 10.1111/j.1469-7580.2008.00953.x.

Drake R, Vogl A, Mitchell A, Tibbitts R, Richardson P. 2015. Gray's atlas of anatomy. Philadelphia, PA: Churchill Livingstone Elsevier.

Druzinsky RE, Doherty AH, De Vree FL. 2011. Mammalian masticatory muscles: homology, nomenclature, and diversification. Integrative and Comparative Biology 51:224-234. DOI: $10.1093 /$ icb/icr067.

Edgeworth F. 1914. On the development and hyoid of the mandibular and hyoid muscles of mammals. Quarterly Journal of Microscopical Science.

Edgeworth FH. 1923. On the development of the cranial muscles of Tatusia and Manis. Journal of anatomy 57:313-35.

Edgeworth FH. 1924. On the masticatory, intermandibular, and hyoid muscles of Orycteropus capensis. Journal of Anatomy 58:134-9.

Endo H, Ito K, Watabe H, Nguyen S, Koyabu D. 2017. Macroscopic and CT examinations of the mastication mechanism in the southern tamandua. Mammal Study 42:89-96. DOI: https://doi.org/10.3106/041.042.0203.

Endo H, Niizawa N, Komiya T, Kawada S, Kimura J. 2007. Three-dimensional CT examination of the mastication system in the giant anteater. Zoological science 24:1005- 
1207

1208

1209

1210

1211

1212

1213

1214

1215

1216

1217

1218

1219

1220

1221

1222

1223

1224

1225

1226

Endo H, Nishiumi I, Kurohmaru M, Nabhitabhata J, Chan-Ard T, Nadee N, Agungpriyono S, Yamada J. 1998. The functional anatomy of the masticatory muscles of the Malayan pangolin, Manis javanica. Mammal Study 23:1-8.

Engelmann G. 1985. The phylogeny of the Xenarthra. In: Montgomery G ed. The evolution and ecology of armadillos, sloths, and vermilinguas. Washington, D.C.: Smithsonian Institution Press, 51-64.

Evans HE, De Lahunta A. 2013. Miller's Anatomy of the Dog. Elsevier Health Sciences.

Fabre P-H, Herrel A, Fitriana Y, Meslin L, Hautier L. 2017. Masticatory muscle architecture in a water-rat from Australasia (Murinae, Hydromys ) and its implication for the evolution of carnivory in rodents. Journal of Anatomy 231:380-397. DOI: 10.1111/joa.12639.

Fearnhead RW, Shute CCD, Bellairs AD. 1955. The temporo-mandibular joint of shrews. Proceedings of the Zoological Society of London 125:795-806. DOI: 10.1111/j.10963642.1955.tb00628.x.

Ferreira-Cardoso S, Delsuc F, Hautier L. 2019. Evolutionary tinkering of the mandibular canal linked to convergent regression of teeth in placental mammals. Current Biology 29:468-475.e3. DOI: 10.1016/J.CUB.2018.12.023.

Ferreira-Cardoso S, Fabre PH, de Thoisy B, Delsuc F, Hautier L. 2020. 3D models related to the publication: "Comparative masticatory myology in anteaters and its implications for interpreting morphological convergence in myrmecophagous placentals." 
1228

1229

1230

1231

1232

1233

1234

1235

1236

1237

1238

1239

1240

1241

1242

1243

1244

1245

1246

1247

Frick H. 1951. Über die Trigeminusmuskulatur und die tiefe Facialismuskulatur von Orycteropus aethiopicus. Zeitschrift für Anatomie und Entwicklungsgeschichte 116:202217. DOI: $10.1007 / \mathrm{BF} 00523542$.

Galton JC. 1869. XXIX. The myology of Cyclothurus didactylus. Annals and Magazine of Natural History 4:244-264. DOI: 10.1080/00222936908696043.

Gans C, de Vree F. 1987. Functional bases of fiber length and angulation in muscle. Journal of morphology 192:63-85. DOI: 10.1002/jmor.1051920106.

Gaudin T, Branham D. 1998. The phylogeny of the Myrmecophagidae (Mammalia, Xenarthra, Vermilingua) and the relationship of Eurotamandua to the Vermilingua. Journal of Mammalian Evolution.

Gaudin TJ, Gaubert P, Billet G, Hautier L, Ferreira-Cardoso S, Wible JR. 2020. Evolution \& morphology. In: Challender, D.W.S., Nash, H., Waterman C ed. Pangolins: Science, Society and Conservation. Cambridge, MA: Academic Press,.

Gaudin TJ, Hicks P, Di Blanco Y. 2018. Myrmecophaga tridactyla (Pilosa: Myrmecophagidae). Mammalian Species 50:1-13. DOI: 10.1093/mspecies/sey001.

Gibb G, Condamine F, Kuch M, Enk J. 2016. Shotgun mitogenomics provides a reference phylogenetic framework and timescale for living xenarthrans. Molecular Biology and Evolution 33:621-642.

Gignac PM, Kley NJ. 2014. Iodine-enhanced micro-CT imaging: methodological refinements for the study of the soft-tissue anatomy of post-embryonic vertebrates. Journal of 
1248

1249

1250

1251

1252

1253

1254

1255

1256

1257

1258

1259

1260

1261

1262

1263

1264

1265

1266

1267

1268

Experimental Zoology Part B: Molecular and Developmental Evolution 322:166-176.

DOI: 10.1002/jez.b.22561.

Ginot S, Claude J, Hautier L. 2018. One skull to rule them all? Descriptive and comparative anatomy of the masticatory apparatus in five mouse species. Journal of Morphology 279:1234-1255. DOI: 10.1002/jmor.20845.

Goździewska-Harłajczuk K, Klećkowska-Nawrot J, Barszcz K. 2018. Macroscopic and microscopic study of the tongue of the aardvark (Orycteropus afer, Orycteropodidae). Tissue and Cell 54:127-138. DOI: 10.1016/J.TICE.2018.08.015.

Gray H. 1995. Anatomy, descriptive and surgical. Bristol: Parragon Book Service Ltd.

Greaves WS. 2012. The mammalian jaw : a mechanical analysis. Cambridge University Press.

Greene EC. 1935. Anatomy of the rat. New York: Hafner Publishing CO.

Le Gros Clark W. 1924. The myology of the tree-shrew (Tupaia minor). Proceedings of the Zoological Society of London:461-497.

Gueldre G, de Vree F. 1990. Biomechanics of the masticatory apparatus of Pteropus giganteus (Megachiroptera). Journal of Zoology 220:311-332. DOI: 10.1111/j.14697998.1990.tb04310.x.

Hall BK, Herring SW. 1990. Paralysis and growth of the musculoskeletal system in the embryonic chick. Journal of Morphology 206:45-56. DOI: 10.1002/jmor.1052060105.

Hallgrímsson B, Lieberman D, Young N, Parsons T, Wat S. 2007. Evolution of covariance in the mammalian skull. Novartis Foundation Symposium 184:164-185. 
1269 Halliday TJD, Dos Reis M, Tamuri AU, Ferguson-Gow H, Yang Z, Goswami A. 2019. Rapid 1270 morphological evolution in placental mammals post-dates the origin of the crown group.

1271 Proceedings of the Royal Society B: Biological Sciences 286. DOI:

$1272 \quad 10.1098 /$ rspb.2018.2418.

1273 Hautier L. 2010. Masticatory muscle architecture in the gundi Ctenodactylus vali (Mammalia, 1274 Rodentia). Mammalia 74:153-162. DOI: 10.1515/MAMM.2010.025.

1275

1276

1277

1278

1279

1280

1281

1282

1283

1284

1285

1286

1287

1288

1289

Hautier L, Lebrun R, Cox PG. 2012. Patterns of covariation in the masticatory apparatus of hystricognathous rodents: implications for evolution and diversification. Journal of Morphology 273:1319-1337. DOI: 10.1002/jmor.20061.

Hayssen V. 2011. Tamandua tetradactyla (Pilosa: Myrmecophagidae). Mammalian Species 43:64-74. DOI: $10.1644 / 875.1$.

Hayssen V, Miranda F, Pasch B. 2012. Cyclopes didactylus (Pilosa: Cyclopedidae). Mammalian Species 44:51-58. DOI: 10.1644/895.1.

Hedrick BP, Yohe L, Vander Linden A, Dávalos LM, Sears K, Sadier A, Rossiter SJ, Davies KTJ, Dumont E. 2018. Assessing Soft-Tissue Shrinkage Estimates in Museum Specimens Imaged With Diffusible Iodine-Based Contrast-Enhanced Computed Tomography (diceCT). Microscopy and Microanalysis 24:284-291. DOI: $10.1017 /$ S1431927618000399.

Herring S. 1993. Formation of the vertebrate face epigenetic and functional influences. American Zoologist 33:472-483. DOI: 10.1093/icb/33.4.472.

Herring SW, Scapino RP. 1973. Physiology of feeding in miniature pigs. Journal of 
1291

1292

1293

1294

Hiiemae K. 1971. The structure and function of the jaw muscles in the rat (Rattus norvegicus L.). III. The mechanics of the muscles. Zoological Journal of the Linnean Society 50:111-132.

Hiiemae K. 2000. Feeding in mammals. San Diego: Academic Press.

Hiiemae K, Houston W. 1971. The structure and function of the jaw muscles in the rat (Rattus norvegicus L.). Zoological Journal of the Linnean Society 50:75-99. DOI: 10.1111/j.1096-3642.1971.tb00752.x.

Hirschfeld S. 1976. A new fossil anteater (Edentata, Mammalia) from Colombia, S . A . and Evolution of the Vermilingua. :419-432.

Humphry G. 1868. On the myology of Orycteropus capensis and Phoca communis. Journal of Anatomy and Physiology 2.

Humphry P. 1869. The myology of the limbs of the Unau, the Aï, the two-toed anteater, and the pangolin. Journal of Anatomy and Physiology 4:17-78.

Hylander W. 2006. Functional anatomy and biomechanics of the masticatory apparatus. In: Temporomandibular Disorders: An Evidenced Based Approach to Diagnosis and Treatment. 3-34.

Hylander WL, Johnson KR, Crompton AW. 1987. Loading patterns and jaw movements during mastication in Macaca fascicularis: A bone-strain, electromyographic, and cineradiographic analysis. American Journal of Physical Anthropology 72:287-314. DOI: 10.1002/ajpa.1330720304. 
1311 Imai M. 1978. The facial muscles of Manis pentadactyla Linne. Okajimas Folia Anatomica 1312 Japonica 55:1-18. DOI: 10.2535/ofaj1936.55.1_1.

1313 Jaskolka MS, Eppley BL, van Aalst JA. 2007. Mandibular coronoid hyperplasia in pediatric 1314 patients. Journal of Craniofacial Surgery 18:849-854. DOI:

1315

1316

1317

1318

1319

1320

1321

1322

1323

1324

1325

1326

1327

1328

1329

1330

1331 10.1097/scs.0b013e3180a772ba.

Jones M. 1997. Character displacement in Australian dasyurid carnivores: size relationships and prey size patterns. Ecology 78:2569-2587. DOI: 10.1890/00129658(1997)078[2569:CDIADC]2.0.CO;2.

Kendall F, McCreary E, Provance P, Rodgers M, Romani W. 1993. Muscles, testing and function: with posture and pain. Lippincott Williams \& Wilkins.

Kist R, Greally E, Peters H. 2007. Derivation of a mouse model for conditional inactivation of Pax9. Genesis 45:460-464. DOI: 10.1002/dvg.20295.

Klaczko J, Sherratt E, Setz EZF. 2016. Are diet preferences associated to skulls shape diversification in xenodontine snakes? PLOS ONE 11:e0148375. DOI: 10.1371/journal.pone.0148375.

Kohli BA, Rowe RJ. 2019. Beyond guilds: the promise of continuous traits for mammalian functional diversity. Journal of Mammalogy 100:285-298. DOI: 10.1093/jmammal/gyz054.

Kuhlhorn F. 1939. Beziehungen zwischen Ernahrungsweise und Bau des Kauapparates bei einigen Giirteltier-und Ameisenbiirenarten. Morphologische Jahrbücher 84:55-85.

Macalister A. 1875. Report on the anatomy of insectivorous Edentates. The Transactions of 
the Irish Academy 25:491-508.

1333

1334

1335

1336

1337

1338

1339

1340

1341

1342

1343

1344

1345

1346

1347

1348

1349

1350

1351

1352

Maestri R, Patterson BD, Fornel R, Monteiro LR, de Freitas TRO. 2016. Diet, bite force and skull morphology in the generalist rodent morphotype. Journal of Evolutionary Biology 29:2191-2204. DOI: 10.1111/jeb.12937.

McGhee GR. 2011. Convergent evolution : limited forms most beautiful. MIT Press.

Meredith RW, Gatesy J, Murphy WJ, Ryder OA, Springer MS. 2009. Molecular decay of the tooth gene enamelin (ENAM) mirrors the loss of enamel in the fossil record of placental mammals. PLoS Genetics 5. DOI: 10.1371/journal.pgen.1000634.

Meredith R, Janečka J, Gatesy J, Ryder O, Fisher CF, Teeling EC, Goodbla A, Eizirik E, Simão TLL, Stadler T. 2011. Impacts of the Cretaceous terrestrial revolution and KPg extinction on mammal diversification. Science 334:521-24. DOI: DOI: 10.1126/science. 1211028 .

Metscher BD. 2009. Micro CT for comparative morphology: simple staining methods allow high-contrast 3D imaging of diverse non-mineralized animal tissues. BMC Physiology 9:1-14. DOI: 10.1186/1472-6793-9-11.

Mills JRE. 1966. The functional occlusion of the teeth of Insectivora. Journal of the Linnean Society of London, Zoology 46:1-25. DOI: 10.1111/j.1096-3642.1966.tb00081.x.

Miranda FR, Casali DM, Perini FA, Machado FA, Santos FR. 2018. Taxonomic review of the genus Cyclopes Gray, 1821 (Xenarthra: Pilosa), with the revalidation and description of new species. Zoological Journal of the Linnean Society 183:687-721. DOI: 10.1093/zoolinnean/zlx079. 
1353 Montgomery G. 1983. Cyclopes didactylus (tapacara, serafin de platanar, silky anteater). In: 1354 Costa Rican natural history. 461-463.

1355 Montgomery G. 1985. Movements, foraging and food habits of the four extant species of 1356 Neotropical vermilinguas (Mammalia; Myrmecophagidae). In: Montgomery G ed. The 1357 evolution and ecology of armadillos, sloths and vermilinguas. Washington, D.C.: 1358 Smithsonian Institution Press, 365-377.

1359 1360

1361

1362 1363 1364 1365 1366

Murphy RA, Beardsley AC. 1974. Mechanical properties of the cat soleus muscle in situ. American Journal of Physiology 227:1008-1013. DOI: 10.1152/ajplegacy.1974.227.5.1008.

Naples V. 1985a. The superficial facial musculature in sloths and vermilinguas (anteaters). In: The evolution and ecology of armadillos, sloths and vermilinguas. 173-189.

Naples VL. 1985b. Form and function of the masticatory musculature in the tree sloths, Bradypus and Choloepus. Journal of Morphology 183:25-50. DOI: 10.1002/jmor.1051830104.

Naples V. 1986. The morphology and function of the hyoid region in the tree sloths, Bradypus and Choloepus. Journal of Mammalogy 67:712-724.

Naples V. 1999. Morphology, evolution and function of feeding in the giant anteater (Myrmecophaga tridactyla). Journal of Zoology 249:19-41.

Navarrete D, Ortega J. 2011. Tamandua mexicana (Pilosa: Myrmecophagidae). Mammalian Species 43:56-63. DOI: 10.1644/874.1.

Nogueira MR, Peracchi AL, Monteiro LR. 2009. Morphological correlates of bite force and 
1374

1375

1376

1377

1378

1379

1380

1381

1382

1383

1384

1385

1386

1387

1388

1389

1390

1391

1392

1393

1394

diet in the skull and mandible of phyllostomid bats. Functional Ecology 23:715-723. DOI: $10.1111 /$ j.1365-2435.2009.01549.x.

Oron U, Crompton AW. 1985. A cineradiographic and electromyographic study of mastication in Tenrec ecaudatus. Journal of Morphology 185:155-182. DOI: 10.1002/jmor.1051850203.

Owen R. 1856. On the anatomy of the great anteater (Myrmaecophaga jubata, Linn.). The Transactions of the Zoological Society of London 4:117-140. DOI: 10.1111/j.14697998.1862.tb08052.x.

Parsons FG. 1896. Myology of rodents. Part II. An account of the myology of the myomorpha, together with a comparison of the muscles of the various suborders of rodents. Proc. Zool. Soc. London 20:159-192.

Patterson B. 1975. The fossil aardvarks (Mammalia: Tubulidentata). Bulletin of the Museum of Comparative Zoology at Harvard College 147.

Patterson B, Segall W, Turnbull WD, Gaudin TJ. 1992. The ear region in Xenarthrans (=Edentata: Mammalia) Part II. Pilosa (Sloths, Anteaters), Palaeanodonts, and a Miscellany. Geology 24.

Pocock RI. 1924. The external characters of the South American Edentates. Proceedings of the Zoological Society of London 94:983-1031. DOI: 10.1111/j.10963642.1924.tb03325.x.

Pouchet G. 1874. Mémoires sur le grand fourmilier (Myrmecophaga jubata, Linné). Paris: Imprimerie de E. Martinet. 
1395 Price SA, Hopkins SSB, Smith KK, Roth VL. 2012. Tempo of trophic evolution and its

1396 impact on mammalian diversification. Proceedings of the National Academy of Sciences

1397 of the United States of America 109:7008-7012. DOI: 10.1073/pnas.1117133109.

1398

Rapp W von. 1852. Anatomische Untersuchungen über die Edentaten. Tübingen.

1399

Redford K. 1987. Ants and termites as food. Current mammalogy.

1400

1401

1402

1403

1404

1405

1406

1407

1408

1409

1410

1411

1412

1413

1414

1415

Reeve ECR. 1940. Relative growth in the snout of anteaters. A study in the application of quantitative methods to systematics. Proceedings of the Zoological Society of London A110:47-80. DOI: 10.1111/j.1469-7998.1940.tb08460.x.

Reiss KZ. 1997. Myology of the feeding apparatus of myrmecophagid anteaters (Xenarthra: Myrmecophagidae). Journal of Mammalian Evolution 4:87-117. DOI: 10.1023/A:1027366129277.

Reiss KZ. 2001. Using phylogenies to study convergence: the case of the ant-eating mammals. American Zoologist 41:507-525. DOI: 10.1093/icb/41.3.507.

Romer AS. 1974. Vertebrate paleontology. Chicago: Chicago University Press.

Rose KR, Emry RJ. 1993. Relationships of Xenarthra, Pholidota, and fossil "Edentates": the morphological evidence. In: Szalay FS, Novacek MJ, McKenna MC eds. Mammal phylogeny: placentals. New York: Springer-Verlag, 81-102.

Rose K, Emry R, Gaudin T, Storch G. 2005. Xenarthra and Pholidota. In: Rose, Kenneth D.; Archibald JD ed. Placental mammals: origin, timing, and relationships of the major extant clades. Baltimore, MD: Johns Hopkins University Press, 106-126.

Rot-Nikcevic I, Reddy T, Downing KJ, Belliveau AC, Hallgrímsson B, Hall BK, Kablar B. 
1416

1417

1418

1419

1420

1421

1422

1423

1424

1425

1426

1427

1428

1429

1430

1431

1432

1433

1434

1435

1436

2006. Myf5 -/- :MyoD -/- amyogenic fetuses reveal the importance of early contraction and static loading by striated muscle in mouse skeletogenesis. Development Genes and Evolution 216:1-9. DOI: 10.1007/s00427-005-0024-9.

Saban R. 1968. Musculature de la tête. In: Grassé P ed. Traité de Zoologie, XVI. Paris: Masson et Cie, 229-472.

Samuels JX. 2009. Cranial morphology and dietary habits of rodents. Zoological Journal of the Linnean Society 156:864-888. DOI: 10.1111/j.1096-3642.2009.00502.x.

Schindelin J, Arganda-Carreras I, Frise E, Kaynig V. 2012. Fiji: an open-source platform for biological-image analysis. Nature.

Sharp AC, Trusler PW. 2015. Morphology of the jaw-closing musculature in the common wombat (Vombatus ursinus) using digital dissection and magnetic resonance imaging. PLoS ONE 10. DOI: 10.1371/journal.pone.0117730.

Sonntag CF. 1925. A monograph of Orycteropus afer. - I. Anatomy except the nervous system, skin, and skeleton. Proceedings of the Zoological Society of London 95:331437. DOI: 10.1111/j.1096-3642.1925.tb01520.x.

Springer MS, Meredith RW, Teeling EC, Murphy WJ. 2013. Technical comment on "The placental mammal ancestor and the post-K-Pg radiation of placentals." Science 341.

Strobbe F, McPeek MA, De Block M, De Meester L, Stoks R. 2009. Survival selection on escape performance and its underlying phenotypic traits: a case of many-to-one mapping. Journal of Evolutionary Biology 22:1172-1182. DOI: 10.1111/j.14209101.2009.01733.x. 
1437 Tavares ALP, Cox TC, Maxson RM, Ford HL, Clouthier DE. 2017. Negative regulation of

1438 endothelin signaling by SIX1 is required for proper maxillary development.

1439 Development 144:2021-2031. DOI: 10.1242/dev.145144.

1440

1441

1442

1443

1444

1445

1446

1447

1448

1449

1450

1451

1452

1453

1454

1455

1456

1457

Taylor AB, Vinyard CJ. 2009. Jaw-muscle fiber architecture in tufted capuchins favors generating relatively large muscle forces without compromising jaw gape. Journal of Human Evolution 57:710-720. DOI: 10.1016/j.jhevol.2009.06.001.

Toldt C. 1905. Der Winkelfortsatz des Unterkiefers beim Menschen und bei den Saugetieren und die Beziehungen der Kaumuskeln zu demselben, Teil. IL Sitzungsberichte der Kaiserlichen Akademie der Wissenschaften, Mathematisch-Naturwissenschaftliche Classe 114:315-476.

Turnbull WD. 1970. Mammalian masticatory apparatus. Fieldiana Geology 18:149-356.

Varrela J. 1990. Effects of attritive diet on craniofacial morphology: a cephalometric analysis of a Finnish skull sample. The European Journal of Orthodontics 12:219-223. DOI: 10.1093/ejo/12.2.219.

Vickerton P, Jarvis J, Jeffery N. 2013. Concentration-dependent specimen shrinkage in iodine-enhanced microCT. Journal of Anatomy 223:185-193. DOI: 10.1111/joa.12068.

Vicq-d'Azyr MF. 1742. Systême anatomique des quadrupèdes. Encyclopédie méthodique:632.

Vizcaíno SF, Paleobiology S, Summer N, Vizcaino SF. 2009. The teeth of the " toothless ": novelties and key innovations in the evolution of xenarthrans. Paleobiology 35:343-366.

Wainwright PC, Alfaro ME, Bolnick DI, Hulsey CD. 2005. Many-to-one mapping of form to 
1458

1459

1460

1461

1462

1463

1464

1465

1466

1467

1468

1469

1470

1471

1472

1473

1474

1475

1476

1477

1478

function: a general principle in organismal design? Integrative and Comparative Biology 45:256-262. DOI: 10.1093/icb/45.2.256.

Weijs W. 1980. Biomechanical models and the analysis of form: a study of the mammalian masticatory apparatus. American Zoologist 20:707-719. DOI: 10.1093/icb/20.4.707.

Wetzel R. 1985. The identification and distribution of recent Xenarthra (=Edentata). In: Montgomery $\mathrm{G}$ ed. The evolution and ecology of armadillos, sloths and vermilinguas. Washington, D.C.: Smithsonian Institution Press, 5-22.

Wille A. 1954. Muscular adaptation of the nectar-eating bats (Subfamily Glossophaginae). Transactions of the Kansas Academy of Science 57:315. DOI: 10.2307/3626058.

Windle B, Parsons FG. 1899. On the myology of the Edentata. Proceedings of the Zoological Society of London 67:314-339. DOI: 10.1111/j.1469-7998.1899.tb06861.x.

Woods CA, Howland EB. 1979. Adaptive radiation of Capromyid rodents: anatomy of the masticatory apparatus. Journal of Mammalogy 60:95-116. DOI: 10.2307/1379762.

Yeh Y-C. 1984. Comparative studies on characteristic structures of sensory and motor mechanisms in the stomatognathic system of the pangolin, Manis aurita (Mammalia) Part 1. Masticatory muscle and their spindle supply in the pangolin. The Journal of the Stomatological Society, Japan 51:674-688. DOI: 10.5357/koubyou.51.674.

Zelditch ML, Wood AR, Bonett RM, Swiderski DL. 2008. Modularity of the rodent mandible: integrating bones, muscles, and teeth. Evolution \& Development 10:756-768. DOI: 10.1111/j.1525-142X.2008.00290.x. 


\section{Table $\mathbf{1}$ (on next page)}

Abbreviations of the illustrated muscles.

This list includes masticatory, facial-masticatory, intermandibular, and hyoid muscles. 
1 Table 1 - Abbreviations of the illustrated muscles. This list include masticatory, facial-masticatory, 2 intermandibular, and hyoid muscles.

\begin{tabular}{|c|c|c|c|}
\hline Muscle & Abbreviation & Muscle & Abbreviation \\
\hline M. masseter profundus & M.m.p. & M. pterygoideus internus pars anterior & pa-M.p.i. \\
\hline M.masseter superficialis & M.m.s. & M. pterygoideus internus pars posterior & pp-M.p.i. \\
\hline M. masseter superficialis pars anterior & pa-M.m.s. & M. mandibuloauricularis & M.ma. \\
\hline M. masseter superficialis pars posterior & pp-M.m.s. & M. buccinatorius pars externa & pe-M.b. \\
\hline M. temporalis superficialis & M.t.s. & M. buccinatorius pars interna & pi-M.b. \\
\hline M. temporalis profundus pars lateralis & pl-M.t.p. & M. intermandibularis anterior & pa-M.mh. \\
\hline M. temporalis profundus pars medialis & pm-M.t.p. & M. mylohyoideus pars anterior & pp-M.mh. \\
\hline M. temporalis superficialis pars zygomatica & pz-M.t.s. & M. mylohyoideus pars posterior & M.ish. \\
\hline M. pterygoideus externus pars superior & ps-M.p.e. & M. interstylohyoideus & M.gh. \\
\hline M. pterygoideus externus pars inferior & pi-M.p.e. & M.p.i. & M. mastostyloideus \\
\hline M. pterygoideus internus & M.mst. \\
\hline
\end{tabular}

3 


\section{Table 2 (on next page)}

Masticatory muscle volumes $\left(\mathrm{mm}^{3}\right.$; white background) and percentages (grey background) obtained from the 3D models of the contrast-enhanced specimens segmentation.

M.t.s. - M. temporalis superficialis; pz-M.t.s. - M. temporalis superficialis pars zygomatica; pm-M.t.p. - M. temporalis profundus pars medialis; pl-M.t.p. - M. temporalis profundus pars lateralis; M.m.p. - M. masseter profundus; M.m.s. - M. masseter superficialis; ps-M.p.e. - M. pterygoideus externus pars superior; pi-M.p.e. - M. pterygoideus externus pars inferior; paM.p.i. - M. pterygoideus internus pars anterior; pp-M.p.i. - M. pterygoideus internus pars posterior. 
1 Table 2 - Masticatory muscle volumes ( $\mathrm{mm}^{3}$; white background) and percentages (grey background) obtained 2 from the 3D models of the contrast-enhanced specimens segmentation. M.t.s. - M. temporalis superficialis; pz3 M.t.s. - M. temporalis superficialis pars zygomatica; pm-M.t.p. - M. temporalis profundus pars medialis; pl-M.t.p. 4 M. temporalis profundus pars lateralis; M.m.p. - M. masseter profundus; M.m.s. - M. masseter superficialis; ps5 M.p.e. - M. pterygoideus externus pars superior; pi-M.p.e. - M. pterygoideus externus pars inferior; pa-M.p.i. - M. 6 pterygoideus internus pars anterior; pp-M.p.i. - M. pterygoideus internus pars posterior.

\begin{tabular}{c|c|c|c|c|c|c|}
\multicolumn{1}{c}{} & \multicolumn{6}{c|}{ Volume in $\mathrm{mm}^{3}$ /Masticatory volume (\%) } \\
\cline { 2 - 7 } Muscles & \multicolumn{2}{c|}{ C. didactylus } & \multicolumn{2}{c|}{ T. tetradactyla } & \multicolumn{2}{c|}{ M. tridactyla } \\
\hline M.t.s. & 139.8 & 42.2 & 575.2 & 20.9 & 1718.1 & 13.5 \\
\cline { 2 - 7 } pz-M.t.s. & 8.0 & 2.4 & 153.9 & 5.6 & 1198.0 & 9.4 \\
\cline { 2 - 7 } pm-M.t.p. & 19.5 & 5.9 & 120.2 & 4.4 & 510.2 & 4.0 \\
\cline { 2 - 7 } pl-M.t.p. & 33.6 & 10.1 & 128.0 & 4.6 & 594.1 & 4.7 \\
\cline { 2 - 7 } M.m.p. & - & - & 307.1 & 11.1 & 1592.6 & 12.5 \\
\cline { 2 - 7 } M.m.s. & 60.4 & 18.3 & 806.1 & 29.2 & 3951.6 & 31.0 \\
\cline { 2 - 7 } ps-M.p.e. & 9.4 & 2.8 & 135.3 & 4.9 & 1013.6 & 7.9 \\
\cline { 2 - 7 } pi-M.p.e. & 14.0 & 4.2 & 59.0 & 2.1 & 489.5 & 3.8 \\
\cline { 2 - 7 } pa-M.p.i. & 46.5 & 14.0 & 227.9 & 8.3 & 851.8 & 6.7 \\
pp-M.p.i. & & & 245.1 & 8.9 & 842.8 & 6.6 \\
\hline Total & 332.3 & 100 & 2757.8 & 100 & 12762.2 & 100 \\
\hline
\end{tabular}

7 


\section{Table 3 (on next page)}

Facial-masticatory muscle volumes ( $\mathrm{mm}^{3}$; white background) and percentages (grey background) obtained from the 3D models of the segmentation of the contrastenhanced specimens

pe-M.b. - M. buccinatorius pars externa; pi-M.b. - M. buccinatorius pars interna; M.ma. - M. mandibuloauricularis. 
1 Table 3 - Facial-masticatory muscle volumes ( $\mathrm{mm}^{3}$; white background) and percentages (grey background)

2 obtained from the 3D models of the segmentation of the contrast-enhanced specimens. pe-M.b. - $M$.

3 buccinatorius pars externa; pi-M.b. - M. buccinatorius pars interna; M.ma. - M. mandibuloauricularis.

\begin{tabular}{c|c|c|c|c|c|c|}
\multicolumn{4}{c}{ Volume in $\mathrm{mm}^{3}$ /facial-mast. volume (\%) } \\
\hline Muscles & \multicolumn{2}{|c|}{ C. didactylus } & \multicolumn{2}{c|}{ T. tetradactyla } & \multicolumn{2}{c|}{ M. tridactyla } \\
\hline pe-M.b. & 17.9 & 25.7 & 371.9 & 27.4 & 990.4 & 15.1 \\
\cline { 2 - 7 } pi-M.b. & 49.9 & 71.6 & 911.1 & 67.2 & 5589.4 & 85.0 \\
\cline { 2 - 7 } M.ma. & 1.94 & 2.8 & 73.7 & 5.4 & NA & - \\
\hline Total & 69.7 & 100 & 1356.7 & 100 & 6579.8 & 100 \\
\hline
\end{tabular}

4 


\section{Table 4 (on next page)}

Proposed homologies for 5 muscles previously described in pangolins (Edgeworth, 1923) and aardvarks (Edgeworth, 1924; Sonntag, 1925; Frick, 1951).

Soontag (1925) did not describe the parts of the M.m. temporalis. * - this could correspond to a M. masseter profundus; ** - included in the "outer part"; *** - not explicit in the text. M.gh. - M. geniohyoideus; pa-M.mh. - M. mylohyoideus pars anterior; pp-M.mh. - M. mylohyoideus pars posterior; M.t.s. - M. temporalis superficialis; pz-M.t.s. - M. temporalis superficialis pars zygomatica; M.t.p. - M. temporalis profundus. 
1 Table 4 - Proposed homologies for 5 muscles previously described in pangolins (Edgeworth, 1923) and 2 aardvarks (Edgeworth, 1924; Sonntag, 1925; Frick, 1951). Soontag (1925) did not describe the parts of the M.m. 3 temporalis. * - this could correspond to a M. masseter profundus; ** - included in the "outer part"; *** - not explicit 4 in the text. M.gh. - M. geniohyoideus; pa-M.mh. - M. mylohyoideus pars anterior; pp-M.mh. - M. mylohyoideus 5 pars posterior; M.t.s. - M. temporalis superficialis; pz-M.t.s. - M. temporalis superficialis pars zygomatica; M.t.p. 6 M. temporalis profundus

\begin{tabular}{|c|c|c|c|}
\hline Edgeworth, 1923 & $\begin{array}{c}\text { Edgeworth, } 1924 \\
\text { Sonntag, 1925 }\end{array}$ & Frick, 1951 & This study \\
\hline digastricus anterior & digastricus posterior & biventer & M. digastricus \\
\hline intermandibularis anterior & digastricus anterior & mylohyoideus & pa-M.mh. \\
\hline intermandibularis posterior & digastricus anterior & mylohyoideus & pp-M.mh. \\
\hline geniohyoideus & intermandibularis & geniohyoideus & M.gh. \\
\hline zygomaticomandibularis* & zygomaticomandibularis & masseter (schicht 3+4) & M. zygomaticomandibularis \\
\hline- & temporalis ant. portion** & pars medialis - temporalis & M.t.s.+pz-M.t.s. \\
\hline- & temporalis post. portion** & pars posterior - temporalis & Absent \\
\hline- & temporalis inner part*** & pars orbitalis - temporalis & M.t.p.
\end{tabular}

7 


\section{Figure 1}

The skull (A) and mandible (B) of Tamandua tetradactyla shown in lateral (up) and ventral (down) views.

The area in green delimits the temporal fossa. The mandible is shown in dorsal (up), medial (middle), and lateral (down) views. Anterior is to the left. Scale bar $10 \mathrm{~mm}$. 


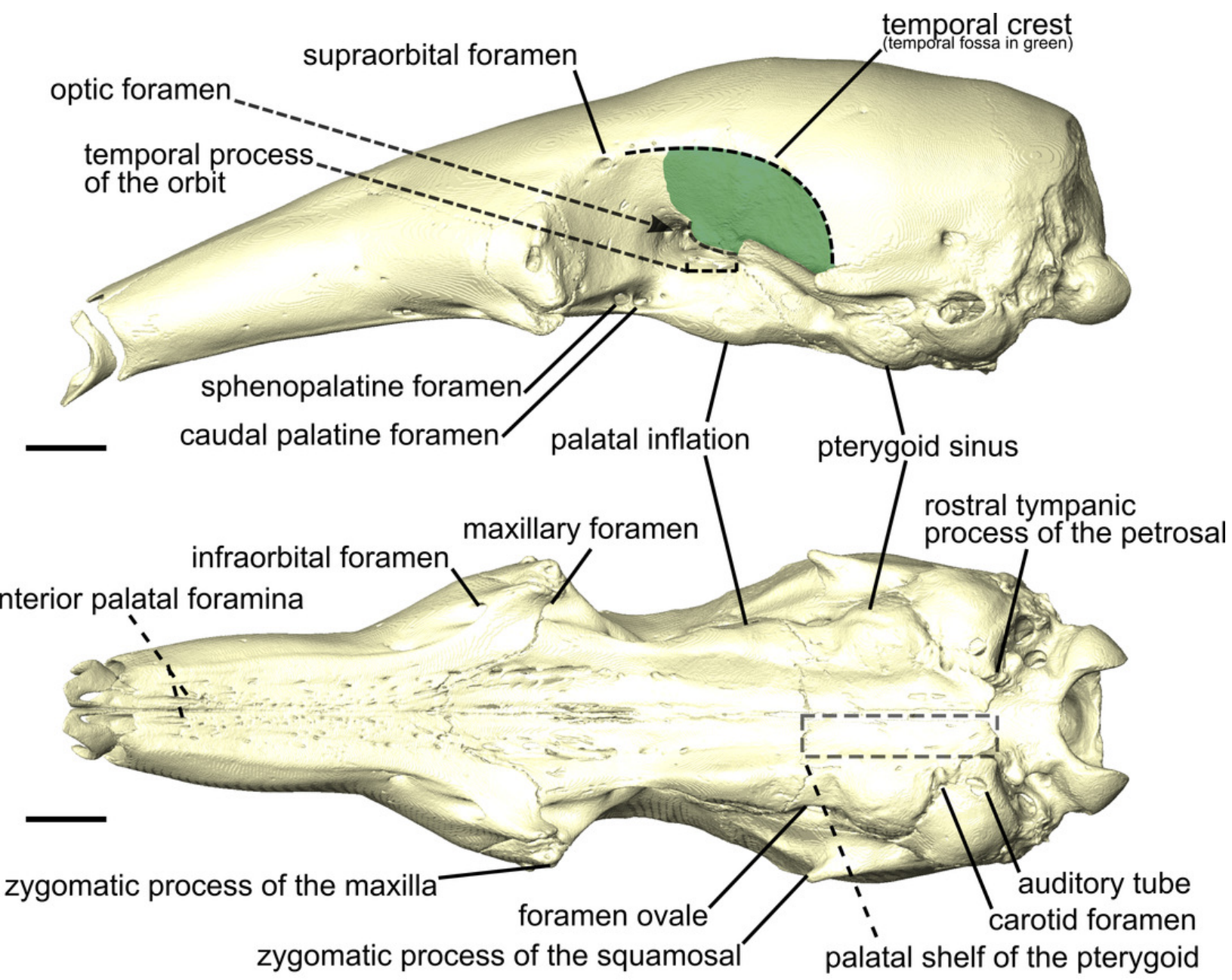

A
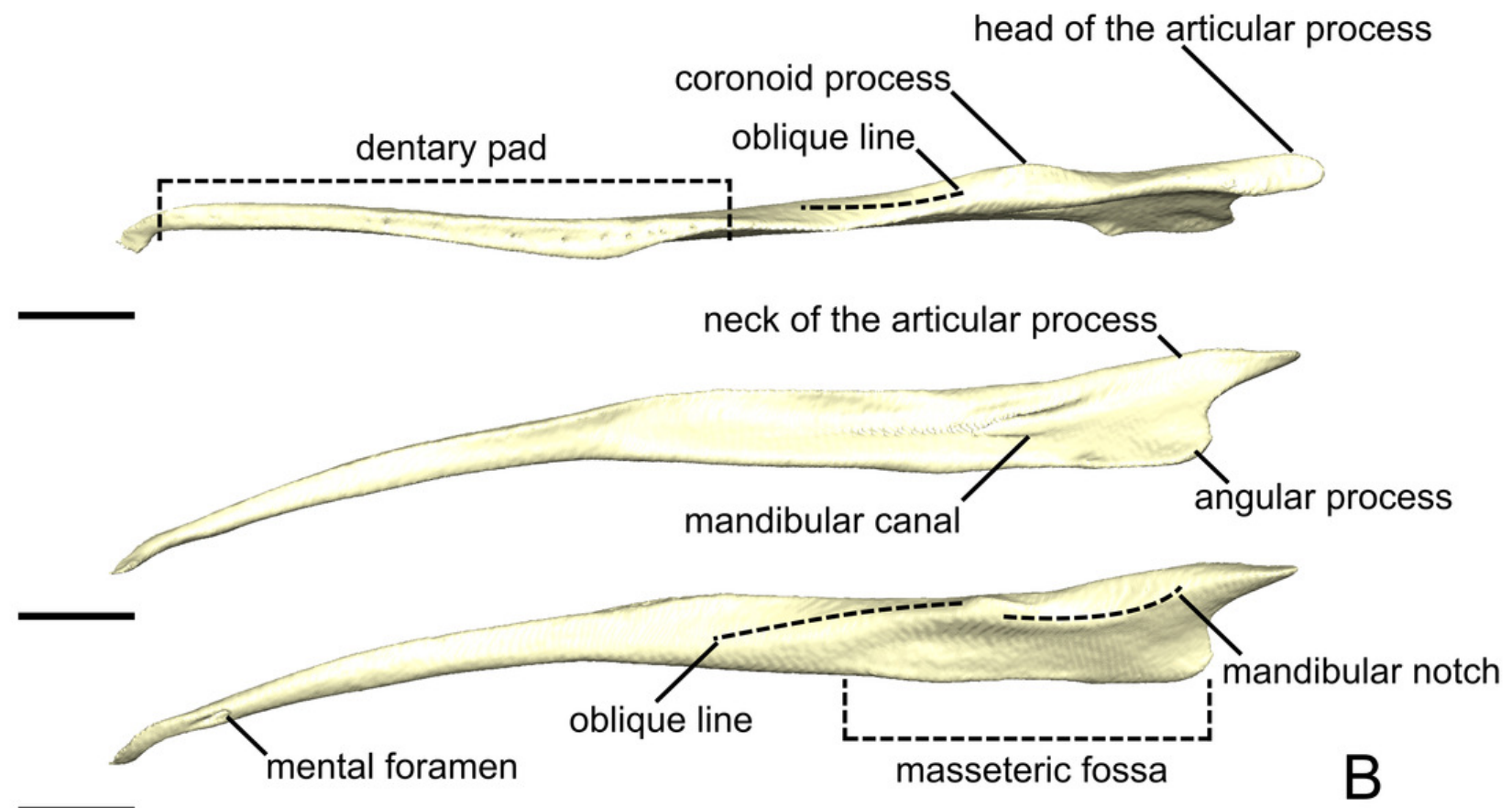
Figure 2

The skull (A, B) and mandible (C, D) of Tamandua tetradactyla shown in lateral (up) and ventral (down) views.

The colored areas represent the origin $(A, B)$ and insertions $(C, D)$ of the masticatory muscles. A legend is provided on the upper-left corner. 
M. temporalis superficialis

M. temporalis superficialis pars zygomatica

M. temporalis profundus pars lateralis

M. temporalis profundus pars medialis

M. masseter superficialis

M. masseter profundus

M. pterygoideus externus pars superior

$\square$ M. pterygoideus externus pars inferior

M. pterygoideus internus pars anterior

M. pterygoideus internus pars posterior
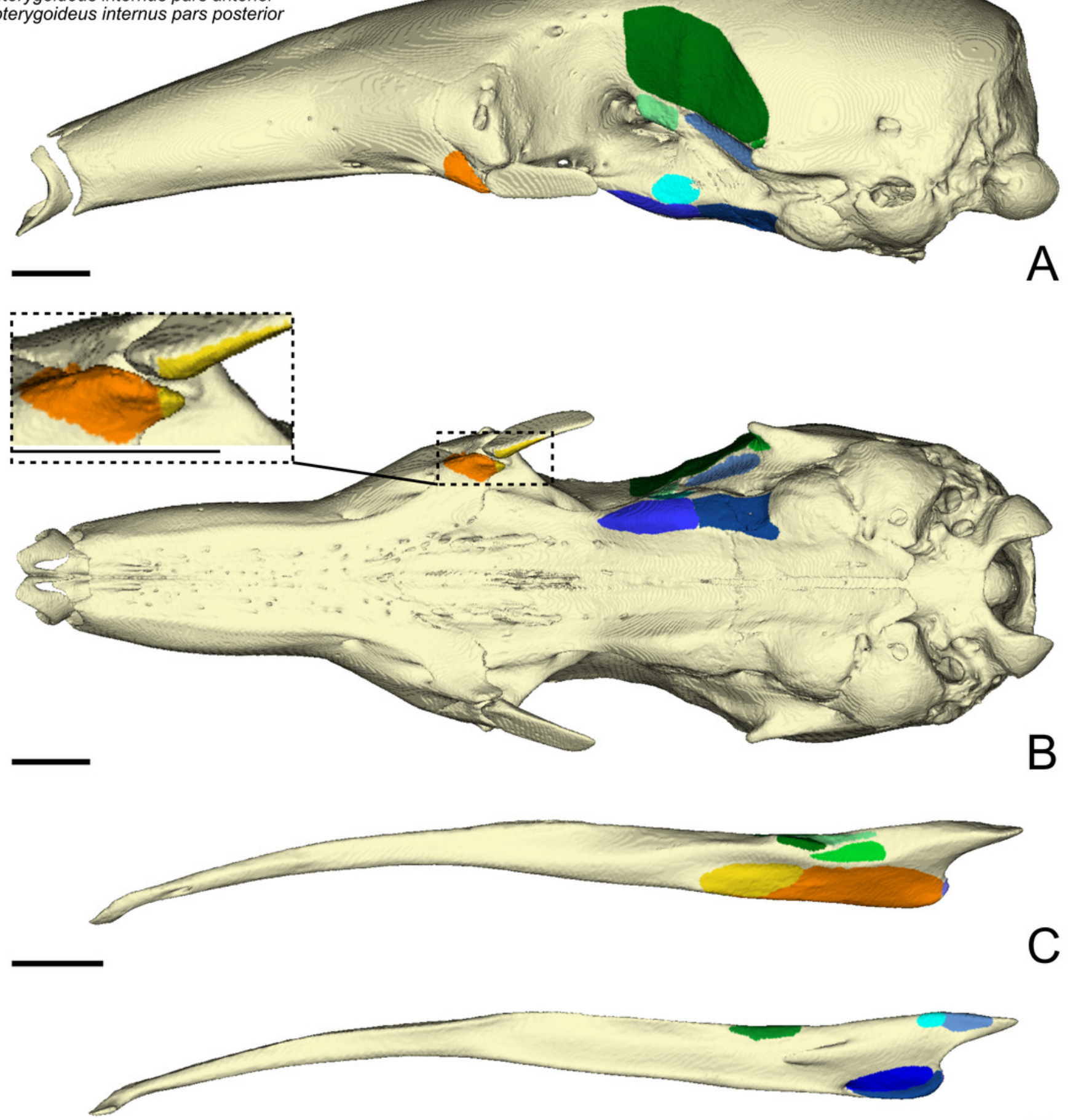

D 
Figure 3

The masticatory and facial-masticatory musculature of Cyclopes didactylus in lateral (A, $B)$, ventral (C), and dorsolateral (D) views.

Scale bar $10 \mathrm{~mm}$. The more superficial muscles were removed in B. Muscle abbreviations as in Table 1. 


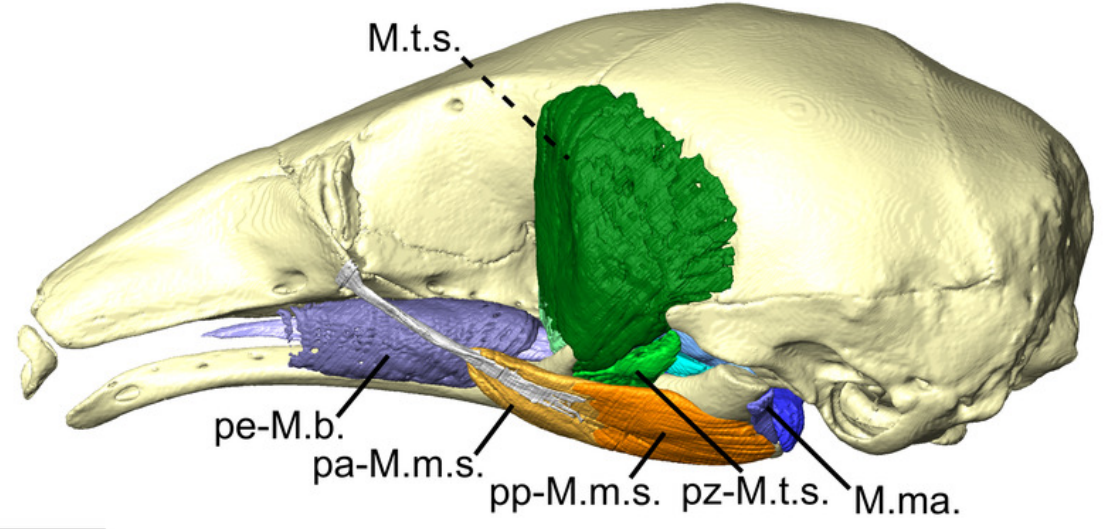

A

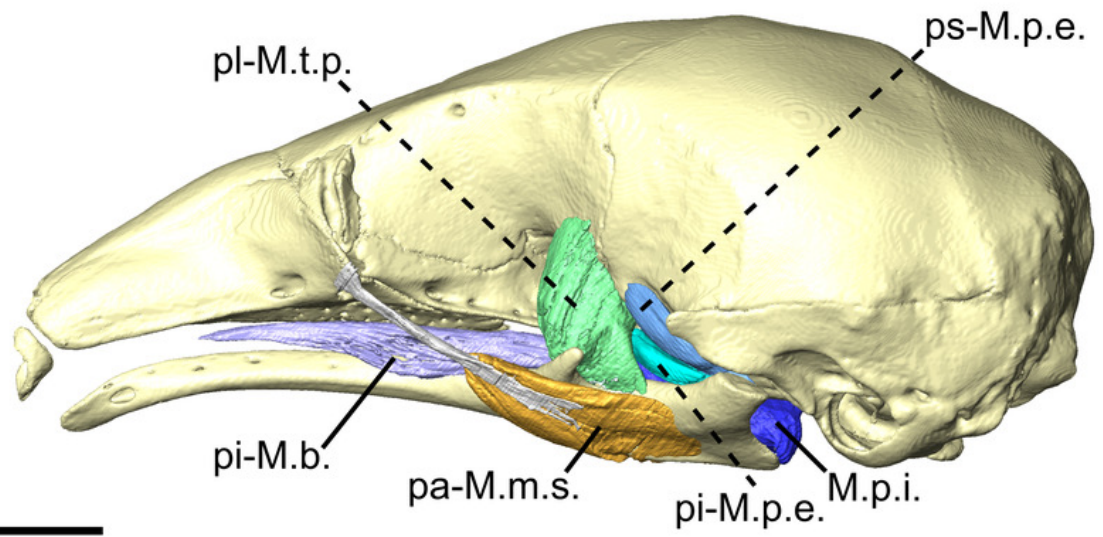

B

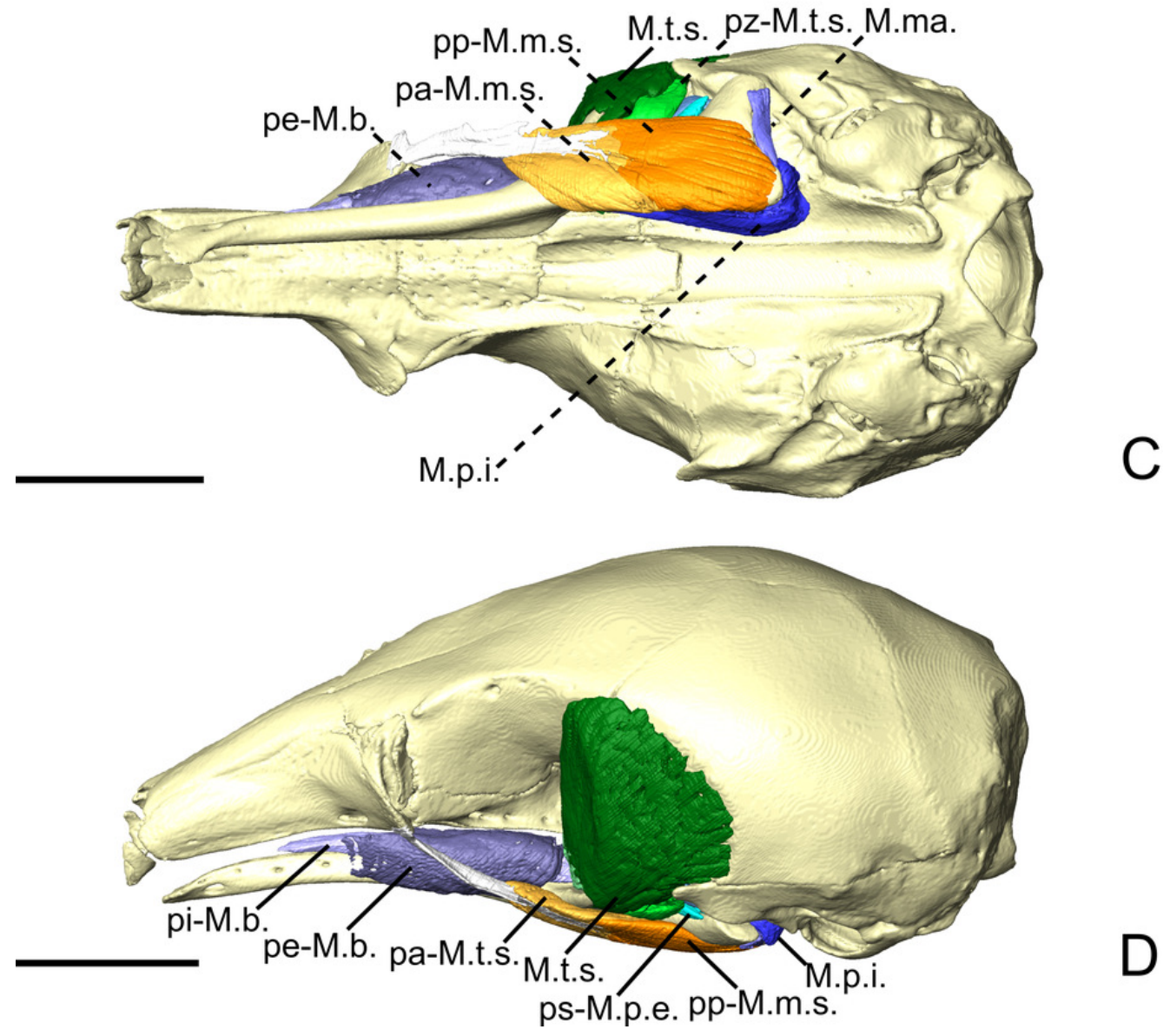


Figure 4

The M. pterygoideus and M. temporalis profundus muscle complexes of $C$. didactylus (A), T. tetradactyla (B), and M. tridactyla in lateral (up) and dorsal (down).

$\mathrm{C}$ is zoomed on the ascending ramus. Scale bar $10 \mathrm{~mm}$. Muscle abbreviations as in Table 1. 

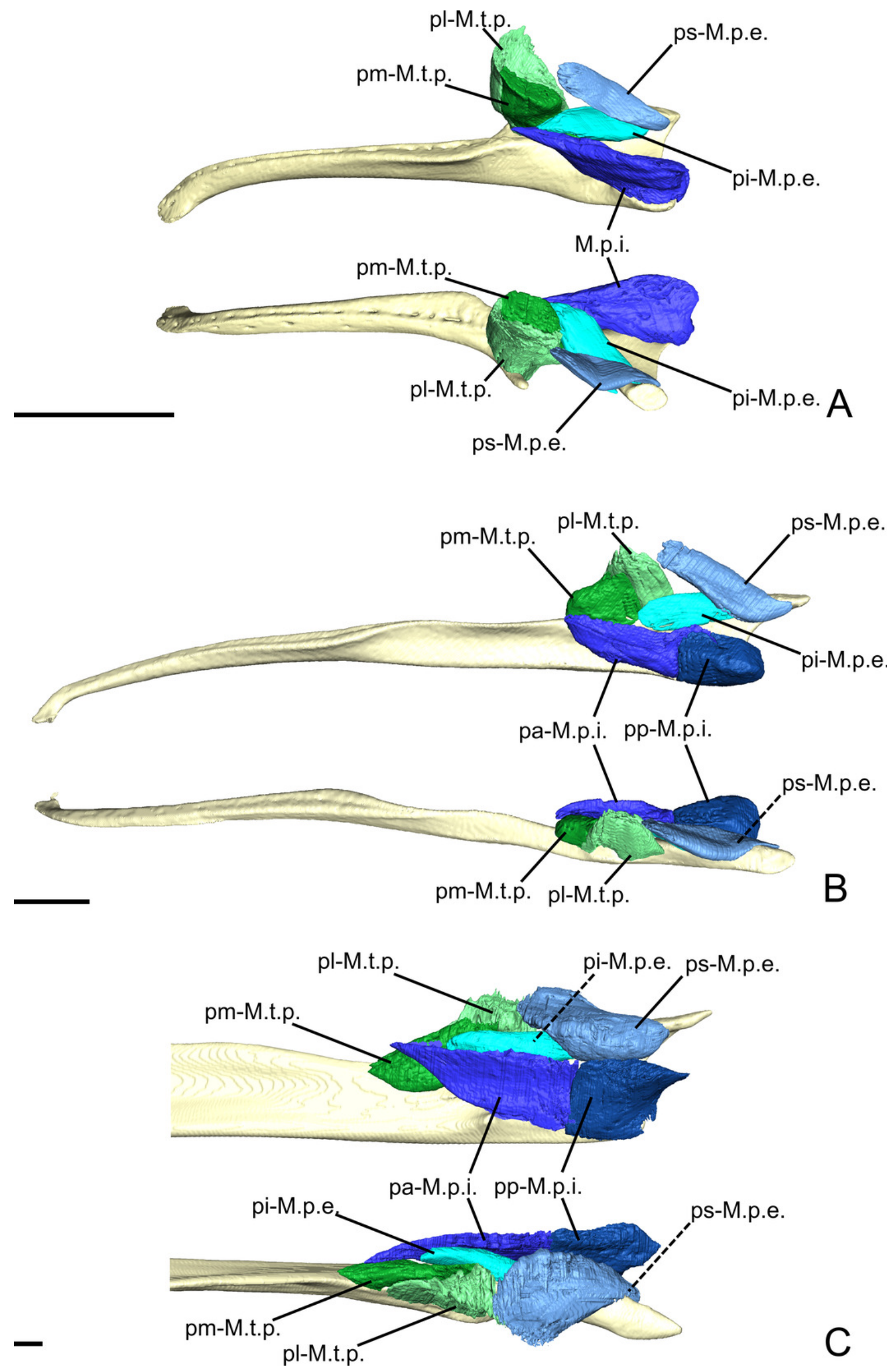

Peer) reviewing PDF | (2020:03:46708:1:1:NEW 26 Jun 2020) 
Figure 5

The intermandibular musculature, M. geniohyoideus, and M. pterygoideus internus of $C$. didactylus in lateral (A), ventral (B), and posteromedial (C) view.

A small vestige of the interstylohyoideus is also depicted. Scale bar $10 \mathrm{~mm}$. Muscle abbreviations as in Table 1. 


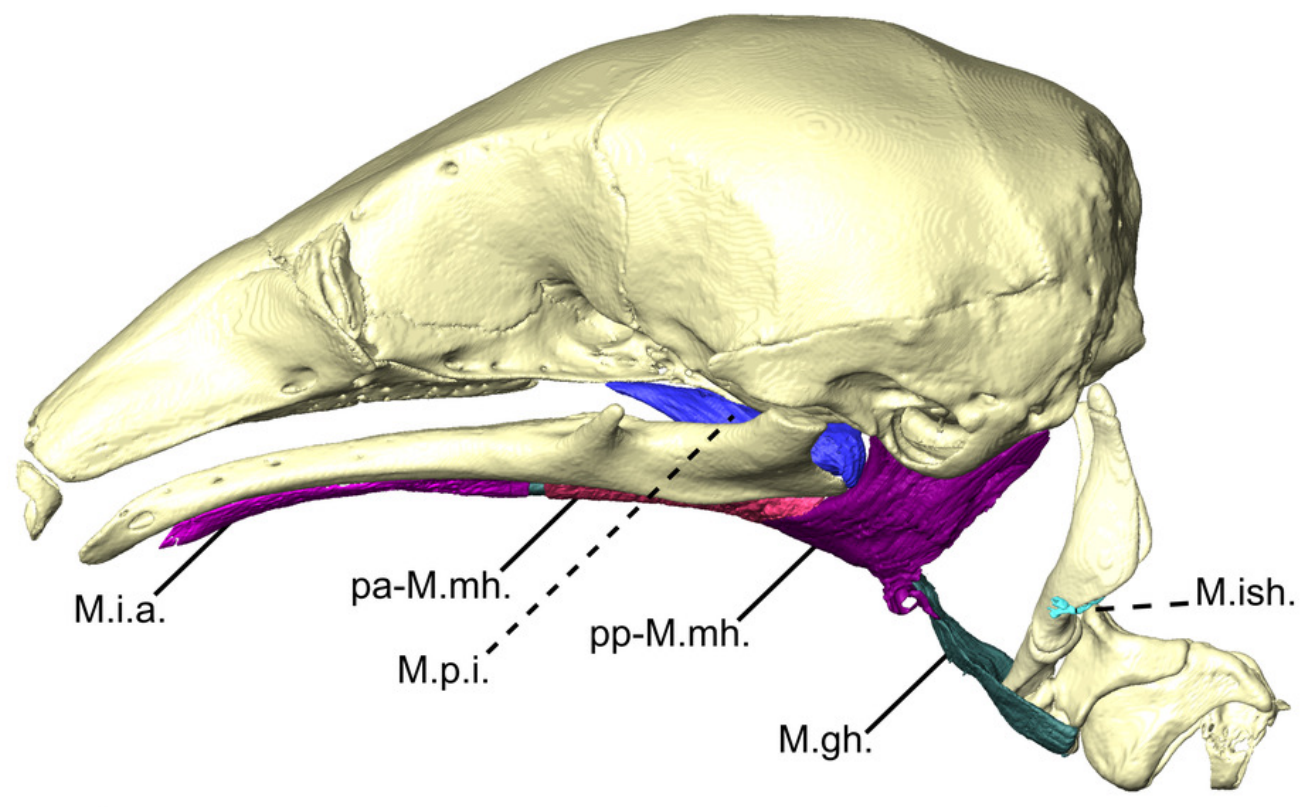

A

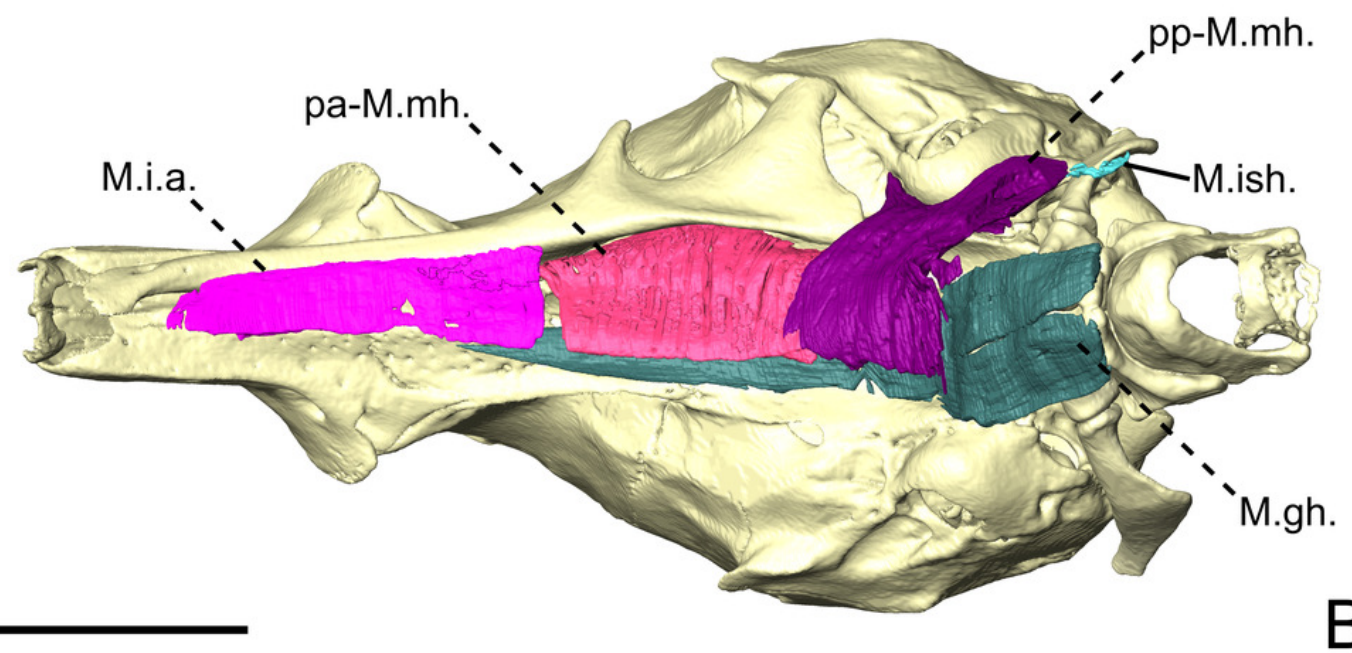

B

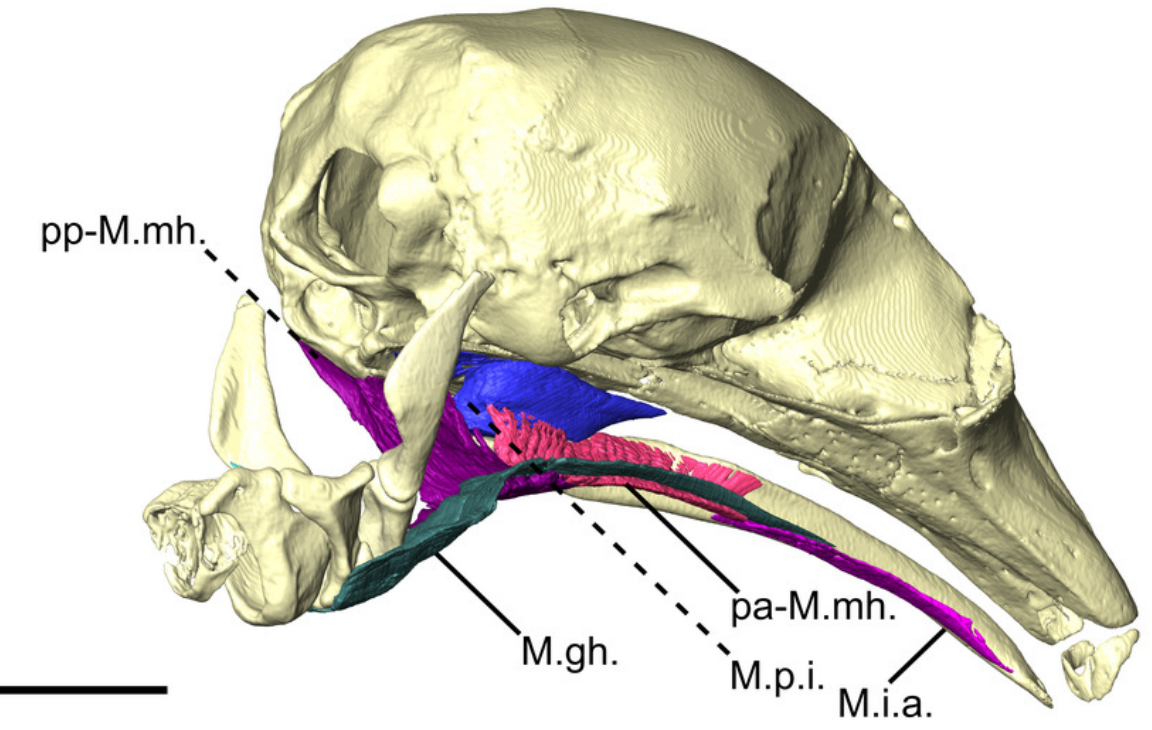


Figure 6

The masticatory and facial-masticatory musculature of $T$. tetradactyla in lateral (A, B), ventral (C), and dorsolateral (D) views. Scale bar $10 \mathrm{~mm}$.

The more superficial muscles were removed in B. Muscle abbreviations as in Table 1. 

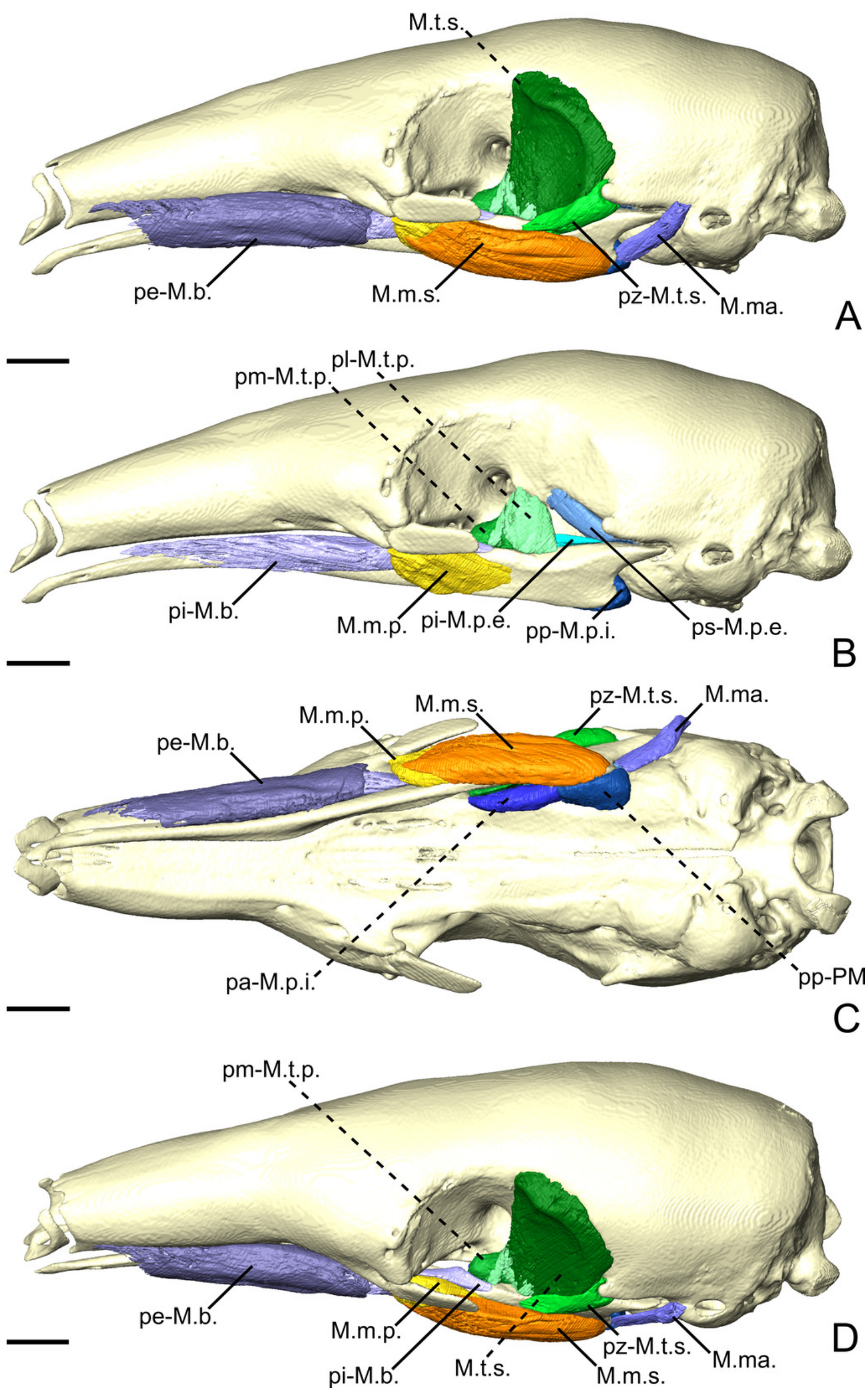

Peer) reviewing PDF | (2020:03:46708:1:1:NEW 26 Jun 2020) 


\section{Figure 7}

The intermandibular musculature, $M$. geniohyoideus, $M$. interstylohyoideus, and $M$. mandibuloauricularis of $T$. tetradactyla in lateral (A), ventral (B), and posteromedial (C) view.

Scale bar $10 \mathrm{~mm}$. Muscle abbreviations as in Table 1. 

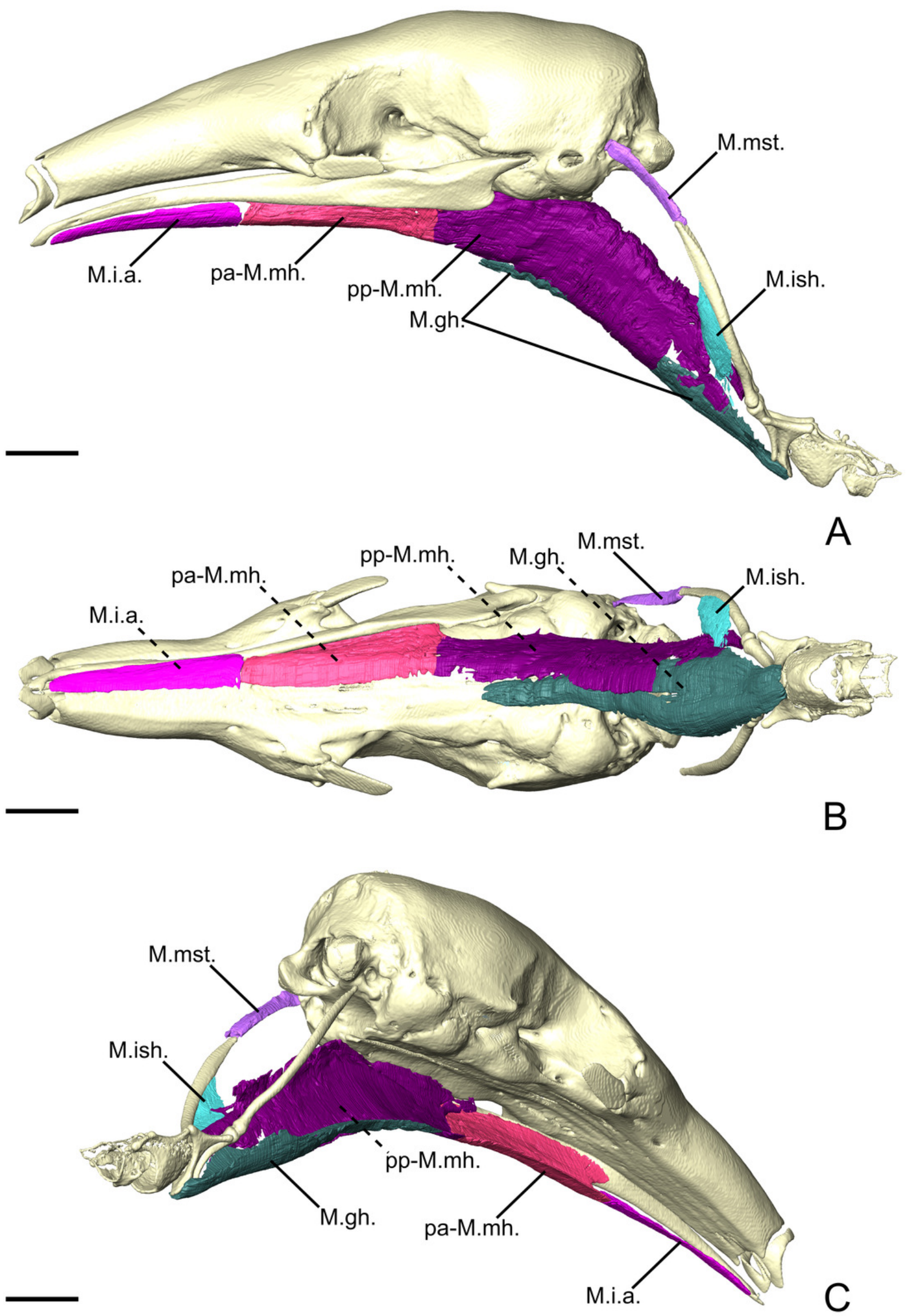
Figure 8

The masticatory and facial-masticatory musculature of $M$. tridactyla in lateral $(A, B)$, ventral (C), and dorsolateral (D) views.

Scale bar $10 \mathrm{~mm}$. The more superficial muscles were removed in B. Muscle abbreviations as in Table 1. 


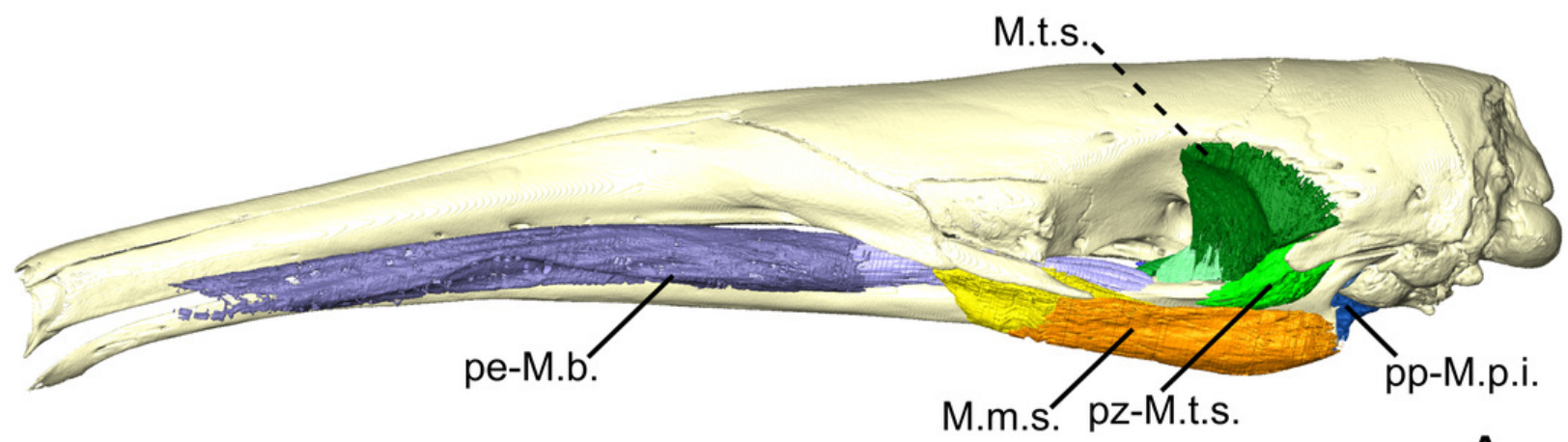

A
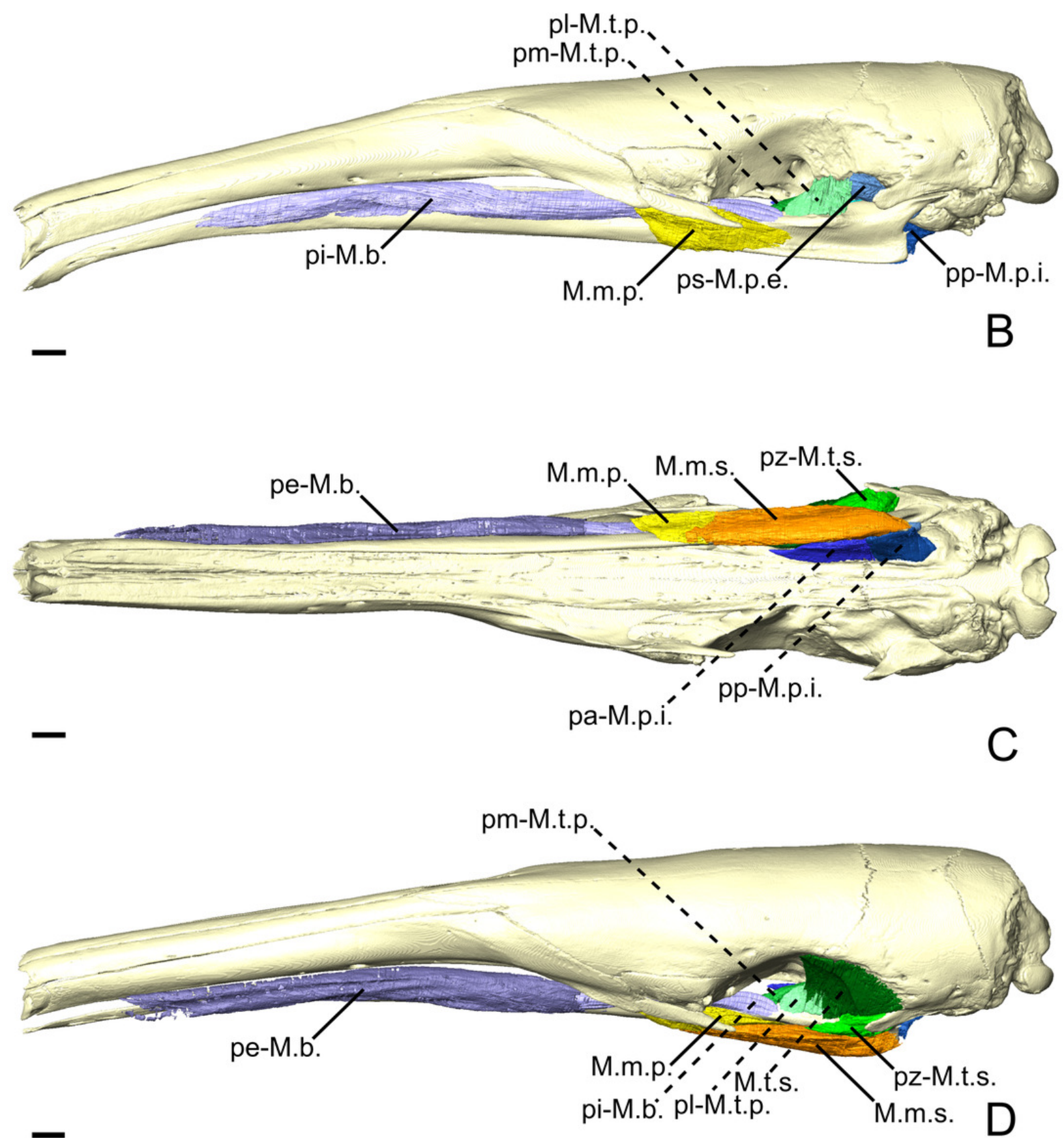
Figure 9

The intermandibular musculature of $M$. tridactyla in lateral (A), ventral (B), and posteromedial (C) view.

Scale bar $10 \mathrm{~mm}$. Muscle abbreviations as in Table 1.

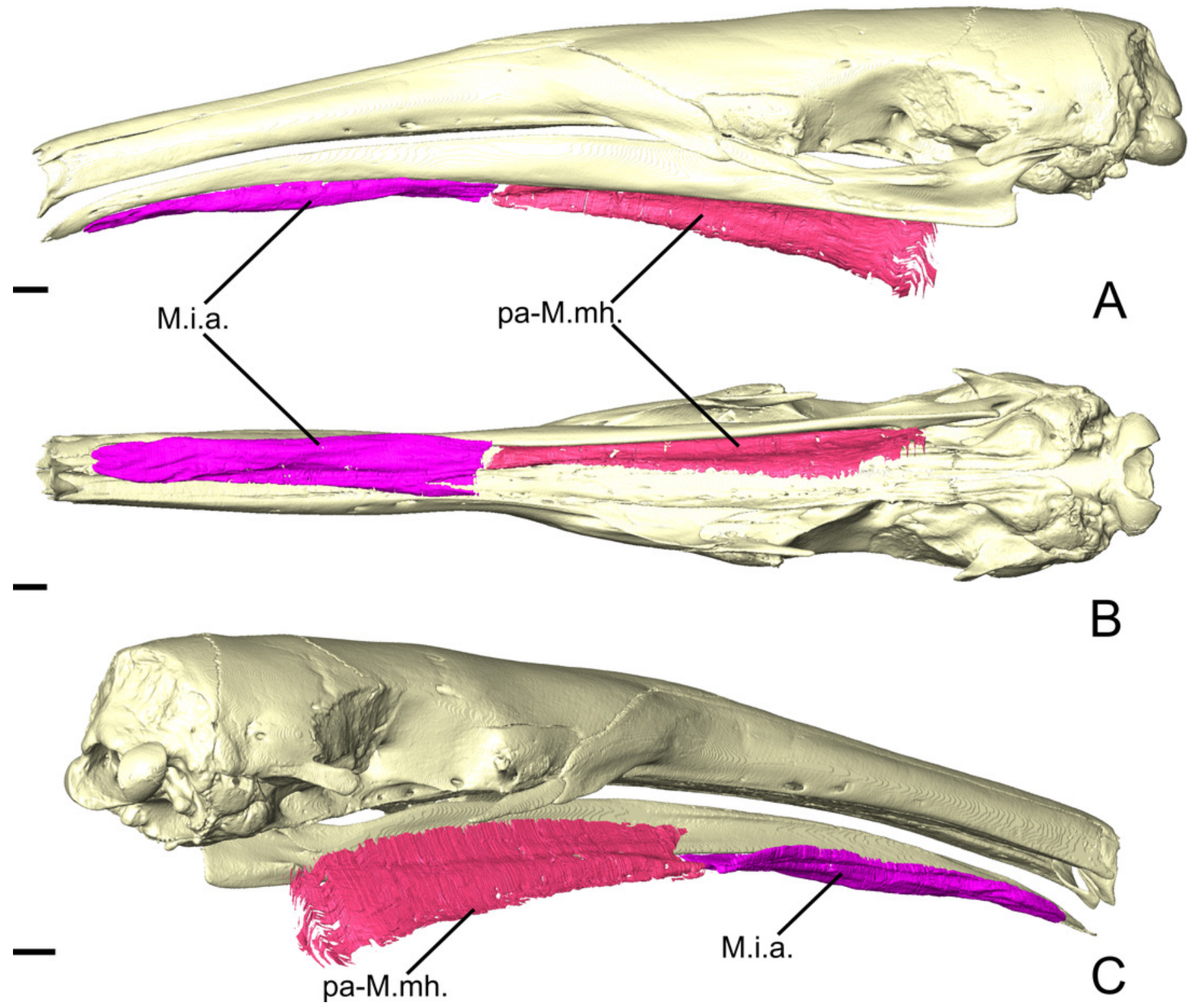




\section{Figure 10}

Mapping of muscular and osteological discrete traits in simplified phylogeny of Pilosa.

Trait 1 refers to the absence of a maxilla-jugal-suqamosal functional unit providing a surface for muscular origins; extant Pilosa all lack completely ossified zygomatic arches, but sloths present strong ligaments connecting the jugal and the zygomatic process of the squamosal from which the zygomaticomandibularis and the masseter profundus arise (Naples, 1985b). Traits 2-11 are based on cranial synapomorphies, directly related to muscular origins/insertions, described in Hirschfeld (1976), Engelmann (1985), and Gaudin \& Braham (1998). The tree was obtained from timetreeoflife.org (Kumar et al., 2017) and divergence times were modified according to Gibb et al. (2016). Silhouettes correspond to one species within the tip taxon. 


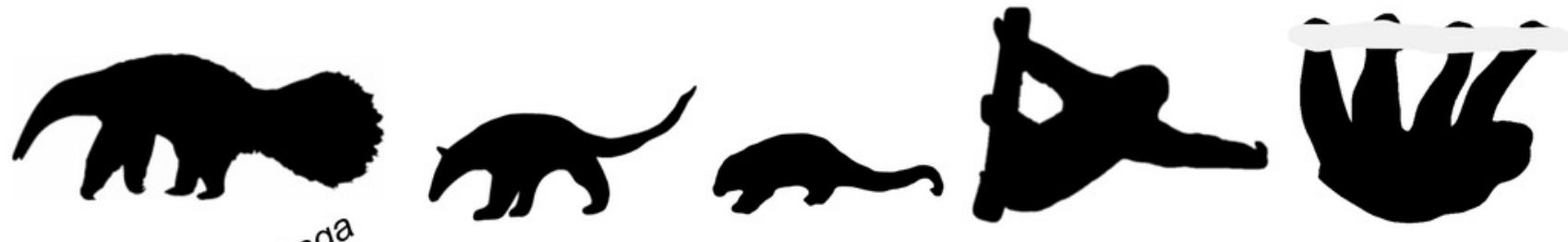

Subdivided M.p.i.

DLoss of M.m.p.

Bipennate fascicles in M.t.s.

$\square$ pa-M.mh. attaches to M.p.i.

$\square$ M.i.a. present

$\square 1$-non-functional zygomatic arch

2-tooth loss

3-unfused symphysis

4-snout elongation

5-pterygoids midline fusion

6 -reduced coronoid process

7-reduced angular process

8-jugal bone loss

9 -concave basicranial/basifacial axis<smiles>[13CH3][13CH3]</smiles><smiles>[13CH3][13CH3]</smiles><smiles>[13CH3][13CH3]</smiles>
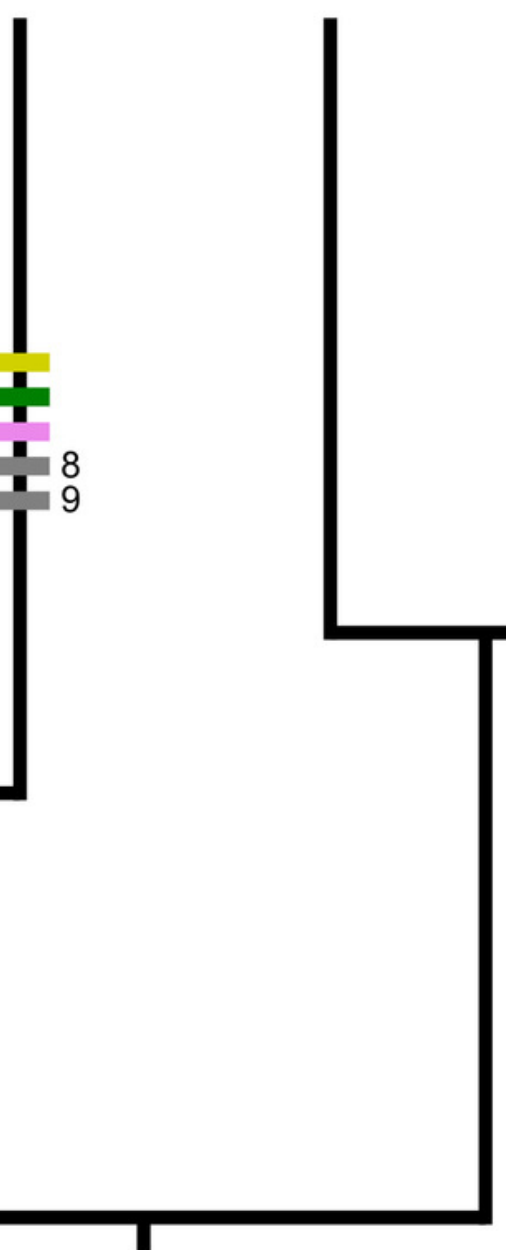
Figure 11

Masticatory and intermandibular muscles lines of action and mandibular dynamics in $T$. tetradactyla (left) and C. didactylus (right).

Lateral views of the skull and mandible with the anteroventral pull directions of the superficial (up) and deep (down) muscles (A, D). Anterodorsal view of the mandibles with the mediolateral component of the lines of action $(B, E)$. Schematic illustration of the mediolateral rotation mandibular movement (Naples, 1999) during mandibular opening (up) and closing (down; C, F). Lines of action color code corresponds to that use for the muscles. Dotted lines represent the lines of action of muscles completely or partially not visible in lateral view. Muscle abbreviations as in Table 1.
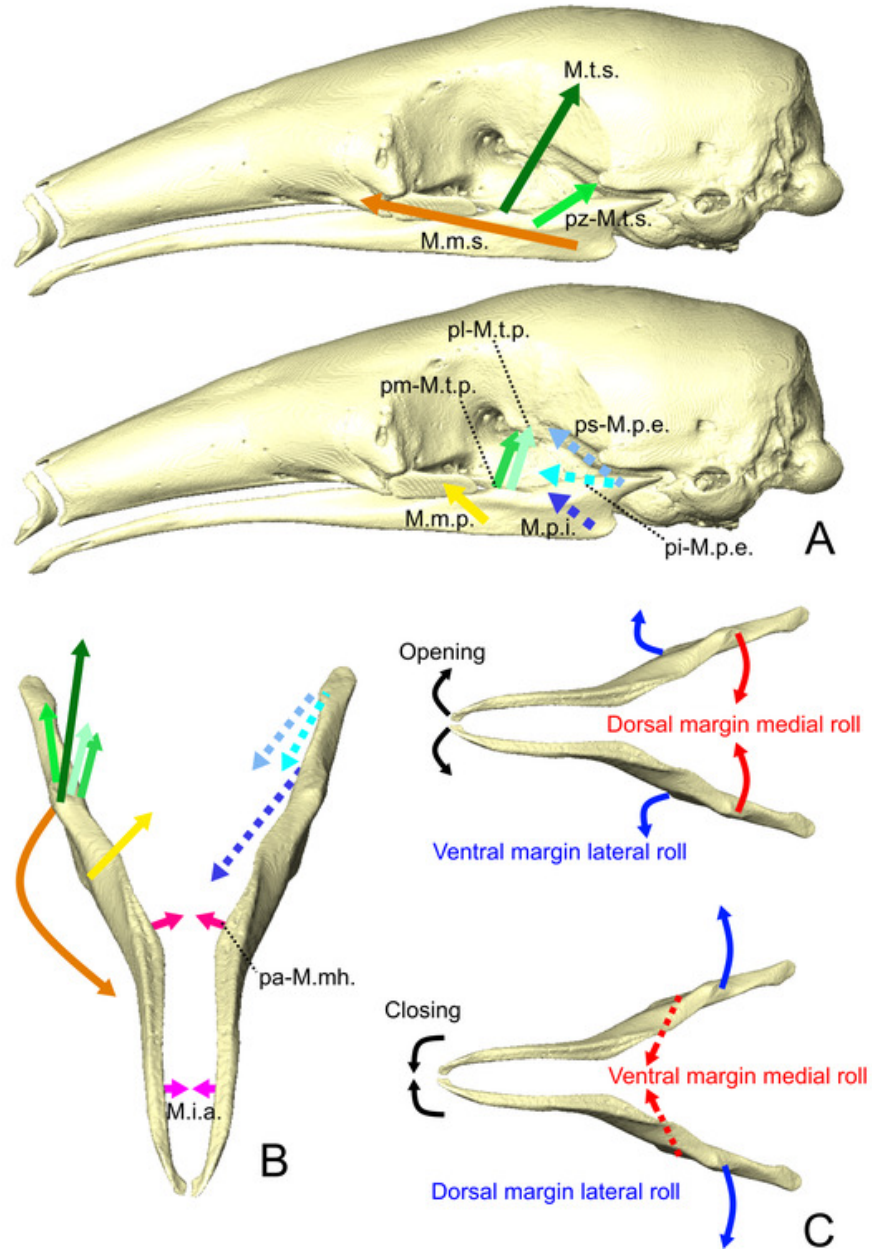
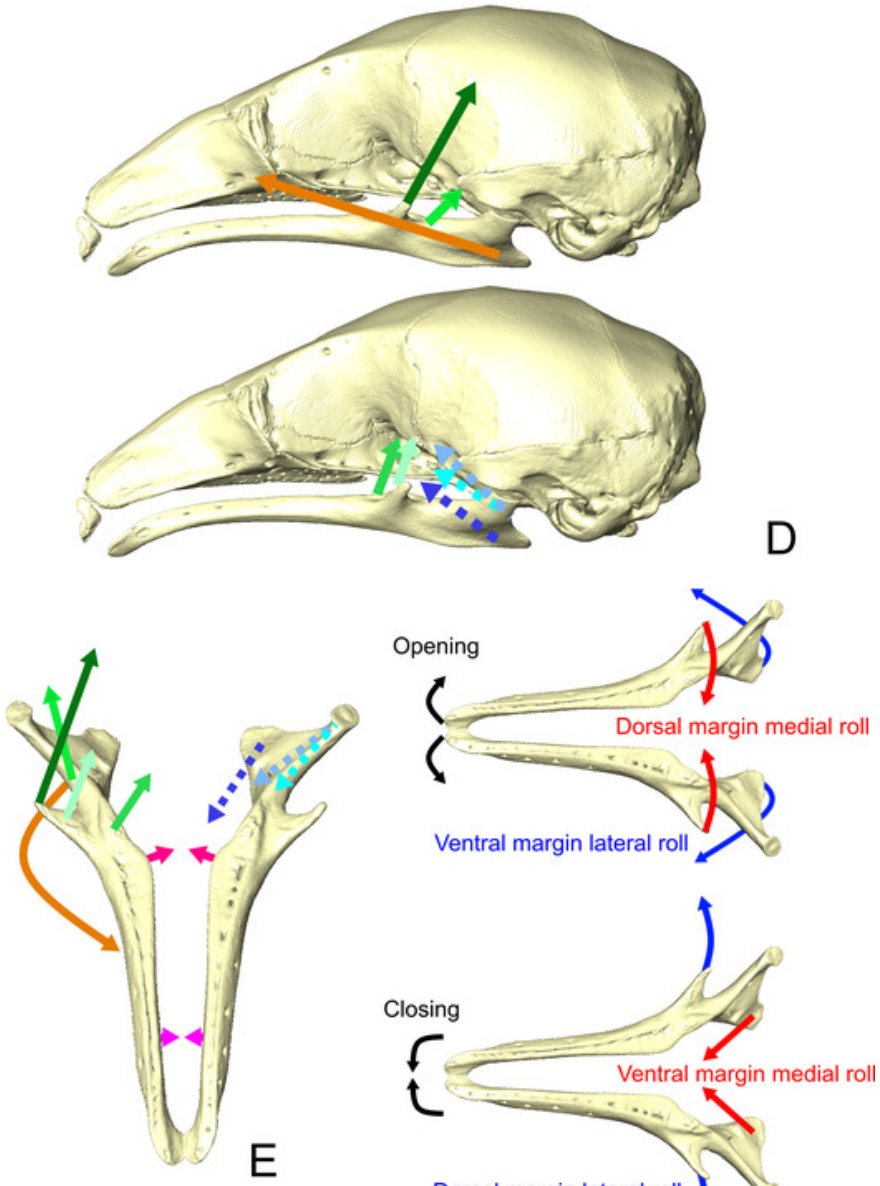

Ventral margin lateral rol

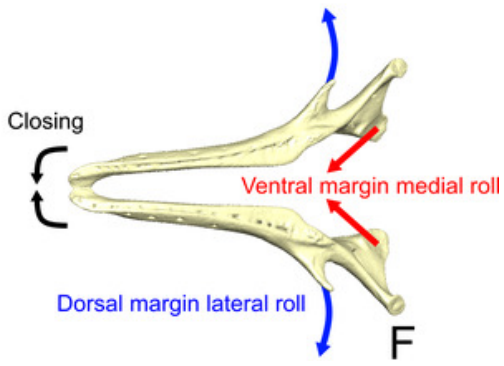

